Portland State University

PDXScholar

Winter 3-20-2015

\title{
Phenotypic and Mutational Consequences of Mitochondrial ETC Genetic Damage
}

\author{
Michael James Lue \\ Portland State University
}

Follow this and additional works at: https://pdxscholar.library.pdx.edu/open_access_etds

Part of the Biology Commons, and the Cell and Developmental Biology Commons Let us know how access to this document benefits you.

\section{Recommended Citation}

Lue, Michael James, "Phenotypic and Mutational Consequences of Mitochondrial ETC Genetic Damage" (2015). Dissertations and Theses. Paper 2202.

https://doi.org/10.15760/etd.2199

This Thesis is brought to you for free and open access. It has been accepted for inclusion in Dissertations and Theses by an authorized administrator of PDXScholar. Please contact us if we can make this document more accessible: pdxscholar@pdx.edu. 
Phenotypic and Mutational Consequences of Mitochondrial ETC Genetic Damage

$$
\text { by }
$$

Michael James Lue

A thesis submitted in partial fulfillment of the requirements for the degree of

\author{
Master of Science \\ in \\ Biology
}

Thesis Committee:

Suzanne Estes, Chair

Kim Brown

Jason Podrabsky

Portland State University

2015 
(C) 2015 Michael James Lue 


\begin{abstract}
Genetic mutation is the ultimate source of new phenotypic variation in populations. The importance of mutation cannot be understated, and constitutes a significant evolutionary force. Although single mutations may have little to no impact on organismal performance or fitness, when multiplied across the total number of potential sites within the genome, mutation can have a large impact. Accurate measurement of the rates, molecular mechanisms, and distributions of effects of mutations are critical for many applications of evolutionary theory. Despite the importance of both deleterious and beneficial mutations, their genome-wide patterns and phenotypic consequences are poorly understood when considering the mitochondrial genome. Mitochondria are organelles that are essential for eukaryotic life. They contain their own genome and generate bioenergy (ATP) necessary to sustain life via the electron transport chain (ETC). Because of their role in eukaryotic physiology, understanding how mitochondrial genetic and phenotypic variation can impact populations and evolutionary outcomes is essential. Past studies have implicated DNA-damaging oxidative stress as a source of mutations within somatic tissue, but there is a gap in knowledge regarding its role in heritable damage within the germ line. In this thesis, I aimed to test this possibility by characterizing the phenotypic and mutational consequences of high intracellular ROS levels caused by mitochondrial ETC genetic damage. I performed experiments using Caenorhabditis elegans ETC mutant, gas-1, and mutation-accumulation (MA) lines generated from this ancestral genotype. I quantified organismal fitness (fecundity and longevity), reactive oxygen species (ROS) levels,
\end{abstract}


mitochondrial membrane potential $(\Delta \Psi \mathrm{M})$, and ATP levels in these lines, and compared the results to those from a set of wildtype control lines. I begin with a general introduction to the hypothesis and the C. elegans system in Chapter I. In Chapter II, I report the findings from this work. In short, I found that while gas-1 MA lines began the experiment with low lifetime fecundity in comparison to the wildtype strain, their fecundity showed no further decline as expected, and even exhibited higher fecundity levels on days 3-5 of reproduction relative to the gas-1 progenitor. The gas-1 progenitor exhibited higher rates of ROS compared to wildtype, whereas the MA lines reverted back to wildtype levels; a similar pattern was observed for $\triangle \Psi \mathrm{M}$, while ATP levels were low in the gas-1 progenitor and remained low in the MA lines. I interpret these findings in light of high-throughput sequencing results from these lines showing that, while nuclear and mitochondrial DNA mutation rates were equal to wildtype in these lines, the genomic pattern of mutation was highly nonrandom and indicative of selection for beneficial or compensatory sequence changes. Because ROS levels declined to wildtype in the evolved (MA), this study was unable to address whether ROS is a major contributor to heritable mutation in this system. I hypothesize that, in addition to their putatively compensatory genetic changes, gas- 1 lineages experienced physiological compensation allowing them to survive, and that this was associated with a "slow living" phenotype. In Chapter III, I summarize general conclusions and implications of this study and end by providing suggestions for further study. 


\section{DEDICATION}

To my friends \& loved ones, who all supported me. From Gregor Mendel, Francis Crick, \& James Watson to Drs. Estes et al., Denver et al., Hassel et al., my wife Krystyna, \& the organisms sacrificed in this study, all of them set up the tenants of the scientific method that I treasure greatly.

"When times get tough, true novelty is needed - novelty whose important features cannot be preplanned - and for this we must rely on chance. Chance is the only source of true novelty."

- Francis Harry Compton Crick, Life Itself: Its Origin and Nature (Crick, 1981) 
TABLE OF CONTENTS

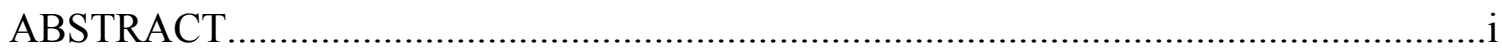

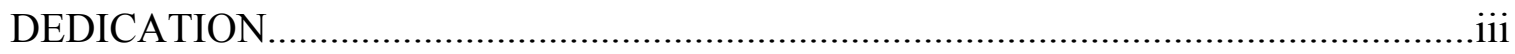

LIST OF TABLES

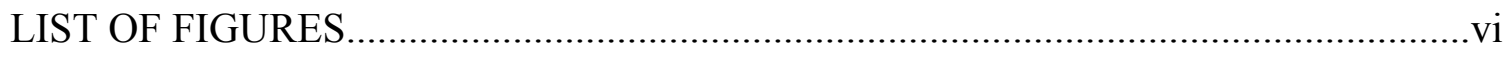

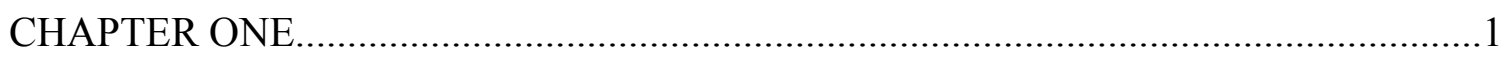

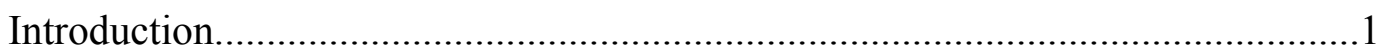

CHAPTER TWO

Phenotypic and mutational consequences of mitochondrial ETC genetic damage..............8

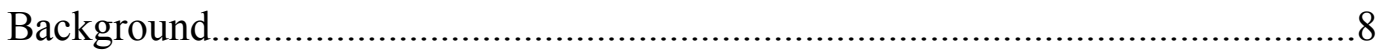

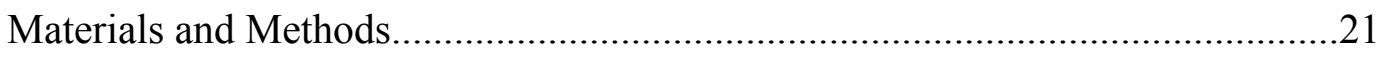

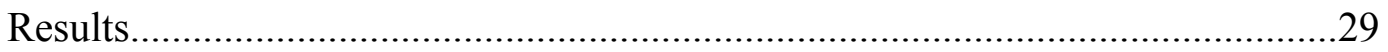

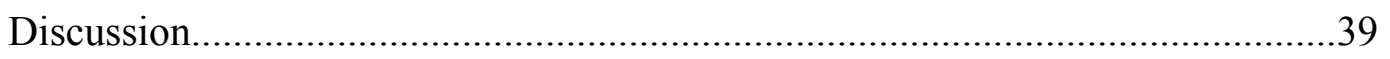

CHAPTER THREE

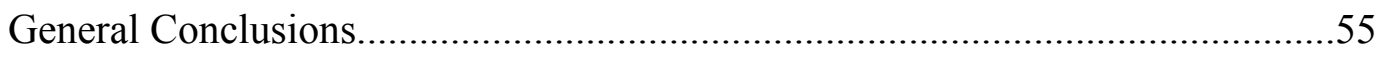

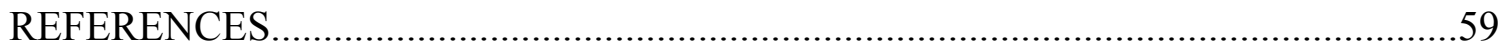




\section{LIST OF TABLES}

Table 1 . Table summary of physiological characteristics among strains........................38 


\section{LIST OF FIGURES}

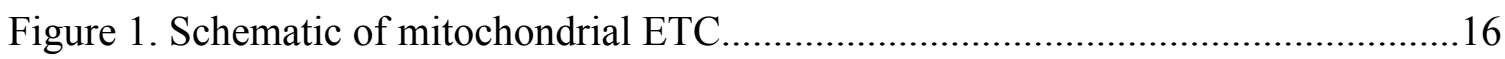

Figure 2. Illustration of the mitochondrial electron transport chain..................................18

Figure 3. Box and whisker plot depicting fecundity in wildtype N2, gas-1 progenitor,

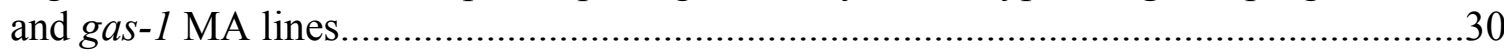

Figure 4. ROS levels of gas-1 progenitor and wildtype N2 ............................................

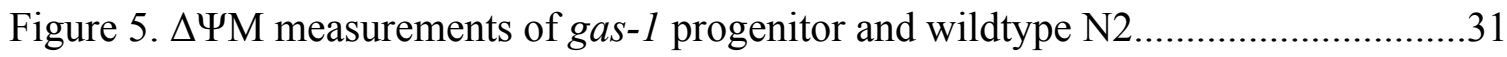

Figure 6. Reproductive schedule on a daily basis of gas-1 MA lines, gas-1 progenitor,

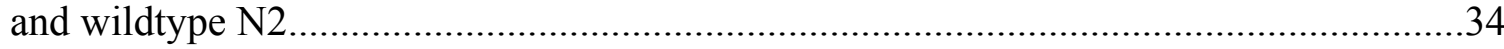

Figure 7. Survival curves of gas-1 MA lines, gas-1 progenitor, and wildtype N2............35

Figure 8. ROS measurements of five gas-1 MA lines, gas-1 progenitor, and

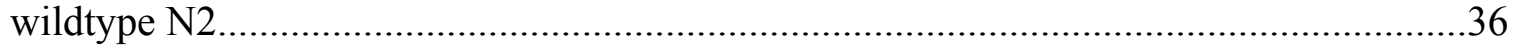

Figure 9. $\Delta \Psi \mathrm{M}$ measurements of five gas-1 MA lines, gas-1 progenitor, and

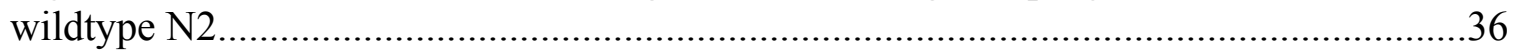

Figure 10. ATP levels of N2 wildtype, gas-1 progenitor, and

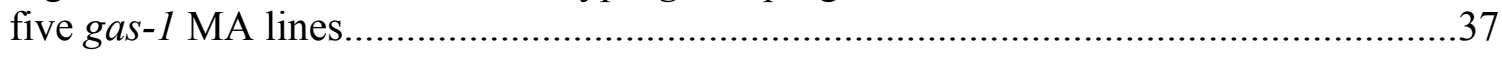

Figure 11. ATP levels of N2 wildtype, gas-1 progenitor, and N2 MA lines.....................39

Figure 12. Pharyngeal pumping rates of five gas-1 MA lines, gas-1 progenitor, and wildtype $\mathrm{N} 2$.

Figure 13. Illumina sequencing results of mtDNA coverage across the mitochondrial genome in gas-1 progenitor and MA lines.....................................................................49

Figure 14. Illustration of the mTOR signaling pathway..............................................52

Figure 15. Illustration of the stress response in a C. elegans cell....................................57 


\section{CHAPTER ONE}

\section{Introduction}

Mutation is a fundamental evolutionary process that underlies genetic change in all populations. Even though mutation is a significantly important factor in the process of evolution, a number of factors give mutation a more subtle nature compared to others. Mutation is typically slower than other evolutionary mechanisms, and the chance a single mutation at an individual site will yield any measureable effect on an organism's phenotype is low. Nonetheless, the large number of sites within a genome implies that most organisms will contain at least one novel mutation not present in the previous generation, regardless of there being an observable impact or not (Schultz \& Lynch, 1997). Mutation therefore constitutes a significant, if subtle, evolutionary force. Newly arising mutations can be detrimental for organismal fitness, although their genome-wide patterns and phenotypic consequences are poorly understood (Burggren \& Freeman, 2011). In eukaryotes, these detrimental mutations can accumulate in both nuclear and mitochondrial genomes (Halligan \& Keightley, 2009; Kondrashov \& Kondrashov, 2010). Mitochondria are organelles that are essential for eukaryotic life. Mitochondria are cellular components that-in addition to containing their own genomes (mtDNA)perform several vital functions including the production of bioenergy in the form of ATP (Ballard \& Whitlock, 2004; Lynch, Koskella, \& Schaack, 2006). Because of their role in eukaryotic physiology, understanding how mitochondrial genetic and phenotypic 
variation can impact populations and evolutionary outcomes is essential. mtDNA genomes appear especially susceptible to mutational damage for several reasons, including their proximity to the site of damaging reactive oxygen species (ROS) production (Dee R Denver et al., 2009).

Nearly all mitochondrial functions depend upon the process of oxidative phosphorylation (OXPHOS) at the mitochondrial electron transport chain (ETC) - a series of protein subunits housed within mitochondrial membranes. The ETC is composed of both nuclearand mitochondrial-encoded protein subunits and its proper functioning depends on the maintenance beneficial interactions between both genomes, known as epistasis (Hicks, Howe, Leung, Denver, \& Estes, 2012). Many features of mitochondrial biology appear to promote high rates of deleterious mutation accumulation in mtDNA compared to the nuclear genome, including: maternal inheritance (small effective clonal interference and genetic drift), many cycles of replication, minimal DNA repair (lack base excision repair) and proximity of damaging oxygen radicals (e.g. ROS) (Ballard \& Whitlock, 2004). The resulting effects may even extend to nuclear DNA (nDNA), affecting a wider range of systems. Because the ETC is crucial for eukaryotic life (L. Azevedo et al., 2009; Dowling, Friberg, \& Lindell, 2008; Harrison \& Burton, 2006; Lane, 2011; Osada \& Akashi, 2011), this genetic system is ideal for studying the patterns, genetic, and phenotypic effects of deleterious mutations. 
One particular mechanism consistently implicated in biological processes such as aging and cancer is oxidative stress (Busuttil, Rubio, Doll, Campisi, \& Vijg, 2003; Yu et al., 2013). Mutations affecting ETC genes can lead to increased ROS levels, which can further damage ETC proteins and, perhaps, mitochondrial and/or nuclear DNA (Lenaz, Fato, et al., 2006). Furthermore, once genomes sustain ROS-induced mutational damage, a "vicious cycle" is hypothesized to occur wherein ROS levels become further elevated and DNA incurs ever more mutational damage, which can lead to aging or heritable disease (Hartman, Ishii, Kayser, Morgan, \& Sedensky, 2001; Raha \& Robinson, 2000; Rand, 2008). Although the consequences of elevated ROS for rates of somatic (nonheritable) mutation have been examined, the extent to which increased mitochondrial ROS may result in heritable mtDNA or nuclear DNA mutations remains unknown (Ameur et al., 2011; Joyner-Matos, Bean, Richardson, Sammeli, \& Baer, 2011).

Although a small fraction of mutations can prove beneficial to organisms, and thus contribute to species' survival and adaptive evolution, the vast majority of newly arising mutations is known to be detrimental for organismal health and fitness. One reason seemingly minute mutations can cause significant debilitation to a population is because mutations of mildly deleterious effect can accumulate within populations, potentially leading to a steady decline of fitness and other phenotypes such as neurological function (Crow \& Kimura, 1970). If multiplied across the total number of potential sites within a genome, the mild effects can become severe; drastically impacting an organism or population. Accurate measures of the rates, molecular mechanisms, and distributions of 
fitness effects of mutation are critical for many applications of evolutionary theory including: inferring evolutionary relationships, testing for selection on molecular sequence, estimating effective population size from standing levels of neutral genetic variation, and parameterizing many population genetic models (Lynch et al., 1999). Despite the importance of deleterious mutations, their genome-wide patterns and phenotypic consequences are poorly understood. Spontaneous mutation is difficult to study due to its rarity in nature, but given the impact that mutation can have on the health and evolutionary trajectories of populations, understanding the rates and properties of spontaneous mutation is a priority (Handel, Regoes, \& Antia, 2006; Jones, Arnold, \& Bürger, 2007; Lande, 1994; Levin \& Walker, 2000; Lynch, Conery, \& Burger, 1995; Lynch \& Walsh, 1997).

One particular method to distinguish the impact of mutation from that of other evolutionary forces affecting rates of molecular evolution and genome content are laboratory mutation accumulation (MA) experiments. MA studies allow mutations to accrue free from selection in the genomes of replicate lineages (MA lines) maintained by self-fertilization or extreme inbreeding across many generations (Halligan \& Keightley, 2009). MA experiments in animals (including Caenorhabditid nematodes) have now been combined with high-throughput sequencing technologies to provide direct estimates of the average per-generation rate of single-nucleotide substitutions and insertions/deletions arising in nuclear (nDNA) genomes along with detailed analyses of their molecular spectra and patterns of bias (Dee R Denver et al., 2009, 2012; Keightley et al., 2009; 
Lynch et al., 2008; Ossowski et al., 2010). Although proving to be a significant tool in elucidating the dynamics of mutation molecular evolution, this approach has not yet been extended to other mutation classes. Evaluations of MA line fitness confirm that most new mutations with phenotypic effects are indeed deleterious (Kondrashov \& Kondrashov, 2010), but the fitness effects of individual mutations are rarely characterized.

Laboratory-conducted experimental evolution allows insight into the mechanistic details of evolution (N Colegrave \& Collins, 2008; Nick Colegrave \& Buckling, 2005; Estes \& Teotonio, 2009). With this technique, comparison of phenotypes and fitness of different populations that have been evolved under particular selective conditions with those of their ancestors is possible. Caenorhabditis elegans nematodes, which are highly fecund, short lived, possess many genetic and genomic resources, and are small enough to manage in large populations under a controlled environment, is currently the best metazoan model organism for using laboratory experimental evolution to understand the genetic mechanisms of evolution. C. elegans are capable of being cryogenically frozen without occurrence of genetic damage, so replicate populations can be propagated for many generations in controlled environments and compared between evolved and ancestral states (R. B. R. Azevedo et al., 2002; Davies, Peters, \& Keightley, 1999; Estes, Ajie, Lynch, \& Phillips, 2005; Estes, Phillips, Denver, Thomas, \& Lynch, 2004; Estes \& Phillips, 2006; Haag-Liautard et al., 2007; Keightley \& Bataillon, 2000; Vassilieva, Hook, Lynch, \& Aug, 2000). 
Caenorhabditid nematodes have also emerged as an important and frequently used model for studying ETC dysfunction and its associated biological consequences. From physiological response to oxidative stress (Joyner-Matos et al., 2013; Kayser, Morgan, Hoppel, \& Sedensky, 2001; Smith, Latta, Denver, \& Estes, 2014) and spontaneous mutation studies (D. R. Denver, 2000; Dee R Denver et al., 2009, 2012; Dee R Denver, Morris, Lynch, \& Thomas, 2004; Estes et al., 2004), the small size, highly differentiated tissues, transparent cuticle, short lifespan, and high fecundity of nematodes make them valuable for multi-generation evolutionary studies. In addition, Caenorhabditids and mammals share similar mitochondrial metabolism and ETC function, meaning that nematode studies can provide insights beyond a single taxon (Westermann, 2010).

Because of its wide use in genetic research, many strains of C. elegans are already developed for experimental purposes. The ETC mutant strain utilized for our experiments was gas-1 (fc21), named for its heightened sensitivity to volatile anesthetics (Gentry, Steele, Sedensky, \& Morgan, 2013). The gas-1 (fc21) allele is a single base-pair substitution within a nDNA gene on the $\mathrm{X}$ chromosome that encodes a central component of ETC complex I. Mutations affecting gas-1 and other complex I components typically lead to elevated ROS levels along with reduced mitochondrial membrane potential ( $\Delta \psi$ M) and ATP production (Lenaz, Baracca, Fato, Genova, \& Solaini, 2006; Lenaz, Fato, et al., 2006). In comparison to the standard laboratory wildtype strain, N2, gas-1 mutants exhibit altered mitochondrial function, reduced and delayed fecundity, and phenotypes and behavior that can be described as "slow living". 
To expand our understanding of ROS and its influence on the genome, I sought to characterize the phenotypic and mutational consequences of mitochondrial ETC genetic damage by quantifying mitochondrial reactive oxygen species (ROS), membrane potential $(\triangle \Psi \mathrm{M})$, fecundity, longevity, ATP levels, and pharyngeal pumping $(\mathrm{PP})$ rates within Caenorhabditis elegans natural isolates and mutation accumulations (MA) lines. To elucidate any potential heritable effects of ROS on mtDNA, I compared the wildtype N2 strain with the gas-1 strain. Specifically, I evaluated the effects of the MA treatment on organismal and organellar health using standard fitness assays and subcellular phenotyping - measuring ROS and $\Delta \psi \mathrm{M}$ levels, and ATP production. This information was combined with other phenotypic data generated by the Estes lab and in collaboration with the Denver Lab at Oregon State University, Illumina genome sequence data was provided to develop a comprehensive view of the effects of ETC damage (and associate oxidative stress) on deleterious mutation accumulation in both nDNA and mtDNA genomes. In Chapter two, I report significant variation in a number of these traits between the gas-1 progenitor and forty gas-1 MA lines compared to wild type control lines. In addition, I discuss these findings in light of evidence of compensatory evolution in C. elegans. Finally, I summarize the broad conclusions and potential impacts of our observations in Chapter three. 


\section{CHAPTER TWO}

\section{Background}

Genetic mutation is the ultimate source of new phenotypic variation in populations. Many evolutionary mechanisms (e.g., directional and stabilizing selection, genetic drift) can lead to a loss of genetic diversity within populations over time. One mechanism that can reverse this decline is mutation. Although slow compared to other evolutionary mechanisms such as genetic drift and natural selection, as well as statistically unlikely when considering mutation rates at individual loci, the large number of loci per genome means that most organisms will contain at least one novel mutation not present in the previous generation (Schultz \& Lynch, 1997). Mutation therefore constitutes a significant, if subtle, evolutionary force. For example, because most new mutations are deleterious for organismal fitness, the impact of spontaneous mutation is thought be a key contributor to important processes such as population extinction risk and the evolution of sex (Burggren \& Freeman, 2011).

Although a small fraction of mutations will prove beneficial to organisms, and thus contribute to adaptive evolution and species' survival, the vast majority of newly arising mutations is known to be detrimental for organismal health and fitness. Mutations of mildly deleterious effect can accumulate within populations, leading to a steady decline

of fitness (Crow \& Kimura, 1970). If multiplied across the total number of potential sites 
within a genome, the mild effects may lead to a significant impact. Accurate measures of the rates, molecular mechanisms, and distributions of fitness effects of mutation are critical for many applications of evolutionary theory including: inferring evolutionary relationships, testing for selection on molecular sequence, estimating effective population size from standing levels of neutral genetic variation, and parameterizing many population genetic models (Lynch et al., 1999). Despite the importance of deleterious mutations, their genome-wide patterns and phenotypic consequences are poorly understood. Spontaneous mutation is difficult to study due to its rarity in nature, but given the impact that mutation can have on the health and evolutionary trajectories of populations, understanding the rates and properties of spontaneous mutation is a priority (Handel et al., 2006; Jones et al., 2007; Lande, 1994; Levin \& Walker, 2000; Lynch et al., 1995; Lynch \& Walsh, 1997).

\section{DNA Damage and Mutation}

DNA genomes are subject to continual damage from various sources; if such damage is neither eliminated nor repaired, it can lead to lasting mutation. DNA damage can be divided into two general categories: endogenous and exogenous. Some endogenous sources of damage include oxidation of bases and strand interruption (e.g., lesions 8oxoguanine and 5-hydroxyuracil) by reactive oxygen species (ROS), alkylation (methylation) of bases, hydrolysis of bases such as deamination, depurination, and depyrimidination, and DNA replication errors. Exogenous sources of damage include 
external factors such as UV-A and UV-B light, leading to direct and indirect DNA damage respectively, ionizing radiation, thermal disruption, and direct chemical damage. With these sources of damage, permanent changes to an organism's DNA, or mutation, can occur if repair mechanisms are lacking or fail altogether. Such mutation can be heritable if it occurs in the germ line. These mutations can cause beneficial, neutral, or deleterious impacts on an organism's fitness (Burggren \& Freeman, 2011).

\section{Mutation-Accumulation (MA) Experimental Approaches}

A primary experimental method for studying heritable mutation has been laboratory mutation-accumulation (MA) experiments, which seek to distinguish the impact of mutation from that of other evolutionary forces affecting rates of molecular evolution and genome content. MA studies allow mutations to accrue free from selection in the genomes of replicate lineages (MA lines) maintained by self-fertilization or extreme inbreeding across many generations (Halligan \& Keightley, 2009). This leads to extreme genetic drift (and minimized natural selection operating on any newly arising mutation), and thereby to an increased likelihood of mutation accumulation. For example, MA studies conducted with Caenorhabditis elegans nematodes, and paired with direct Sanger sequencing of select nuclear genome sequences, showed that $C$. elegans mutants deficient in mismatch repair experienced higher rates of heritable base-substitution mutation than did mutants deficient in other repair pathways, consistent with this pathway being most 
important for repairing this form of DNA damage and maintaining genetic health (Dee R Denver, Feinberg, Steding, Durbin, \& Lynch, 2006).

MA experiments in animals (including Caenorhabditid nematodes) have now been combined with high-throughput sequencing technologies to provide direct estimates of the average per-generation rate of single-nucleotide substitutions and indels (insertions or deletions) arising in the entire nuclear (nDNA) genome along with detailed analyses of their molecular spectra and patterns of bias (Denver et al., 2009, 2012; Keightley et al., 2009; Lynch et al., 2008; Ossowski et al., 2010). For instance, Saxer et al. conducted an MA experiment with the amoeba Dictyostelium discoideum and used high-throughput sequencing to find lower nDNA and mtDNA mutation rates compared to multicellular taxa (Saxer et al., 2012). Furthermore, the nDNA mutation rates were the lowest reported in all eukaryotes. The researchers suggest a more effective DNA-repair mechanism and mutation resistance in comparison to multicellular eukaryotes along with natural genetic adaptation. More recently, Heilbron et al. utilized MA experiments in the bacterium Pseudomonas aeruginosa to determine a significant amount of the resulting fitness decay was due to the fixation of highly deleterious and rare mutations comprising $0.05 \%$ of all fixed mutations observed (Heilbron, Toll-Riera, Kojadinovic, \& MacLean, 2014). After an average of 644 generations, the researchers also found that a small fraction, $0.64 \%$, of the mutations discovered via whole-genome sequencing were likely beneficial and fixed due to positive selection - even in the face of extreme genetic drift. Of the 944 mutations recorded amongst all 8 strains, the majority was base substitutions (>99\% were 
transitions) followed by indels (insertions were 2.5-fold larger than deletions). Heilbron et al. demonstrated that fitness decay in P. aeruginosa is highly influenced by rare mutations of large, deleterious effect. This result is consistent with other evaluations of MA line fitness, confirming that most new mutations with phenotypic effects are indeed deleterious (Kondrashov \& Kondrashov, 2010), although the fitness effects of individual mutations are rarely characterized.

\section{Mutation in Nuclear and Mitochondrial Genomes}

In eukaryotic cells, mutations occur in both nuclear (nDNA) and mitochondrial (mtDNA) genomes, which, in humans, consist of approximately three million kilobase pairs, encoding around 20,000-25,000 genes (Consortium, 2004), and around 17 kilobase pairs (38 genes) (Schaefer, Taylor, Turnbull, \& Chinnery, 2004), respectively. Apart from the dramatic difference in genome size, differences in the population biology of the nucleus and mitochondria (e.g., biparental versus uniparental inheritance) generate differences in the mutational process within each genome. Variation in number of factors, such as DNA replication activity, exposure to mutagens, DNA repair capability and activity, and mutational buffering by the presence of particular genetic pathways have been implicated in generating mutation rate variation between nDNA and mtDNA genomes and across species (Baer, Miyamoto, \& Denver, 2007). 
Studies by the C. F. Baer and D. R. Denver groups provide the informational basis for the current study. In 2005, Baer et al. sought to elucidate whether baseline rates and properties of mutation varied between taxa (Baer et al., 2005). The researchers conducted MA experiments in the relative absence of natural selection in three species of nematode from the family Rhabditidae: C. elegans, C. briggsae, and Oscheius myriophila. Two replicate, highly inbred (genetically homogeneous) strains from each species were subjected to an average of 200 generations of MA and were assessed for fitness changes in parallel with the ancestral controls. The Bateman-Mukai method was applied to these data to estimate mutation rates and average effects of new mutations (Baer et al., 2005). The authors concluded that substantial genetic variation in the mutation processes existed between genera and replicate lineages (i.e., only the two C. elegans strains did not significantly differ from each other). The researchers also estimated the per-generation rates of decline in mean fitness to be similar across generations of MA. One particular lineage of O. myriophila did not decline in fitness over the 200 generations of MA, a pattern the authors suggested could result from acquisition of compensatory mutation. Lastly, Baer et al. report average Bateman-Mukai estimates of mutation rate to be twice as large in $C$. briggsae compared to the two $C$. elegans strains, potentially indicating that genomic mutation rate (or average mutational effect) in C. briggsae is twice as high, providing strong evidence that genomic mutational properties are different between taxa.

Denver et al. have applied DNA sequencing approaches to nematode MA lines to characterize mutation rates and the underlying genetic, genomic, and evolutionary causes 
of variation in mutational properties (Dee R Denver et al., 2004; Lemire, 2005). Using a different set of C. elegans MA lines (Vassilieva et al., 2000; Vassilieva \& Lynch, 1999) Denver et al. (Dee R Denver et al., 2004) used Sanger sequencing of diverse nDNA regions to provide the first direct and unbiased estimate of the nuclear mutation rate and spectrum in an animal. In particular, Denver et al. found that base substitutions were four times as frequent as indels, transitions were more common than transversions, and frameshift-causing indel mutations were biased toward insertions.

Denver et al. also characterized heritable base-substitution and indel mutation in the mitochondrial genome by directly sequencing mtDNA from the above C. elegans MA lines (Lemire, 2005). Located within mitochondria are multiple copies of the circular mtDNA, which — in C. elegans — contains approximately 14 kilobase pairs (36 genes) (Lemire, 2005). mtDNA may be especially susceptible to deleterious mutation accumulation due to the fact that they are DNA-repair poor (e.g., appear to lack nucleotide excision repair) and are maternally inherited, which together can lead to increased mutation rates and a reduced effective population (i.e., increased genetic drift). Denver et al. analyzed 771,672 bp across 74 independently evolved MA lines to estimate a mutation rate of 14.3 per site per million years (My), where base substitutions occurred with a frequency of 8.9 per site per My. The observed rate was two orders of magnitude higher than indirect phylogenetic estimates. Furthermore, Denver et al. confirmed previous results finding that animal mtDNA evolution was biased towards transitions 
( $\mathrm{G} \Leftrightarrow \mathrm{A}, \mathrm{T} \Leftrightarrow \mathrm{C}$ ), a pattern the researchers concluded was largely reflective of the baseline mutation pattern since little selection occurred in the MA lines (D. R. Denver, 2000).

Denver et al. later applied next-generation sequencing to characterize the same mutation types in a subset of the aforementioned Baer C. elegans MA lines (Dee R Denver et al., 2009). Specifically, the researchers utilized these lines to obtain genome-wide estimates of spontaneous base-substitution mutations processes in nDNA using both highthroughput sequencing-by-synthesis and pyro-sequencing. In so doing, the researchers were able to provide an order of magnitude more mutation data than previous MA-line mutation surveys. They found that mutations were transversion predominant compared to natural patterns of polymorphism in C. elegans - which are transition predominantchallenging previous assumptions concerning spontaneous mutation processes. Additionally, although the proximate cause of DNA damage was unknown, the pattern of mutational bias was indicative of oxidative DNA damage in that the two most common base-substitutions were $\mathrm{G}: \mathrm{C} \rightarrow \mathrm{A}: \mathrm{T}$ transitions $\mathrm{G}: \mathrm{C} \rightarrow \mathrm{T}: \mathrm{A}$ transversions, which are commonly caused by oxidative compounds 5-hydroxyuracil and 8-oxoguanine, respectively. Collaboration with the Denver lab (Oregon State University) has provided the opportunity to work with the same MA lines used in this study and utilize the DNA sequence data in my analyses. 
Mitochondrial Electron Transport Chain (ETC) and Reactive Oxygen Species (ROS)

Located in the mitochondrial inner membrane are multiple protein "supercomplexes" called electron transport chains (ETC). The purpose of the ETC is to convert nutrients consumed by organisms into energy in the form of adenosine triphosphate (ATP). The mitochondrial ETC is composed of both nuclear- and mitochondrial-encoded protein subunits and its proper functioning depends on the maintenance of favorable mitonuclear genetic interactions, or epistasis (Fig. 1, (Hicks et al., 2012)).

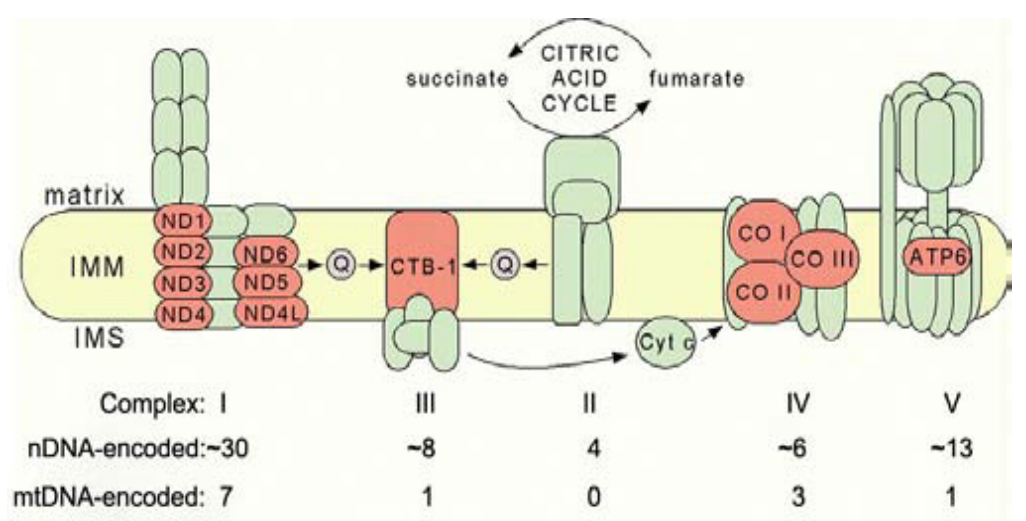

Figure 1. Schematic of mitochondrial ETC. Pink $=$ mtDNA-encoded subunits; green $=n D N A$-encoded subunits. The estimated numbers of subunits comprising the $C$. elegans complexes are listed below. IMM $=$ inner mitochondrial membrane; IMS = intermembrane space; $\mathrm{Q}=$ ubiquinone; Cyt $\mathrm{c}=$ cytochrome $\mathrm{c}$ (Hicks et al., 2012).

Many features of mitochondrial biology appear to promote high rates of deleterious mutation accumulation in mtDNA compared to the nuclear genome, including: maternal inheritance (small effective clonal interference and genetic drift), many cycles of replication, minimal DNA repair (lack nucleotide excision repair and possibly base excision repair) and proximity of damaging oxygen radicals (higher baseline mutation 
rate (Ballard \& Whitlock, 2004)). Because the ETC is crucial for eukaryotic life (L. Azevedo et al., 2009; Dowling et al., 2008; Harrison \& Burton, 2006; Lane, 2011; Osada \& Akashi, 2011), this genetic system is ideal for studying the patterns and functional bases of deleterious mutations occurring in both nuclear and mitochondrial genomes.

Reactive oxygen species (ROS) are highly reactive molecules (Hancock, Desikan, \& Neill, 2001) that are a standard byproduct of cellular respiration. They are involved in cellular functions such as signaling and cellular maintenance. If ROS are allowed to reach high levels, they can damage cellular components including DNA. If ROS production outpaces the detoxification or repair of ROS-induced damage, the result is "oxidative stress". For example, a common ROS byproduct of respiration is superoxide $\left(\mathrm{O}_{2}{ }^{-}\right)$, which can become hydrogen peroxide and damage cell structures or even DNA if not destroyed via an enzyme (e.g. catalase). ROS typically builds up near complex I in the mitochondrial matrix (Fig. 2). Complex I, encoded by both nDNA and mtDNA (Fig. 1), is the largest, least well known complex estimated to be at around $1000 \mathrm{kD}$ in mammals (Kayser et al., 2001). ETC complex I serves as a main entry site for electrons into the oxidative phosphorylation system and initiates their transfer from NADH to ubiquinone through FMN (flavin mononucleotide) and iron-sulfur clusters (Hirst, Carroll, Fearnley, Shannon, \& Walker, 2003). These redox reactions are coupled to the pumping of protons across the mitochondrial inner membrane, helping to generate the electrochemical gradient $(\Delta \psi \mathrm{M})$ that fuels ATP production. Occasional leakage of electrons onto 
molecular oxygen makes this complex the primary site of ROS production (Raha \& Robinson, 2000).

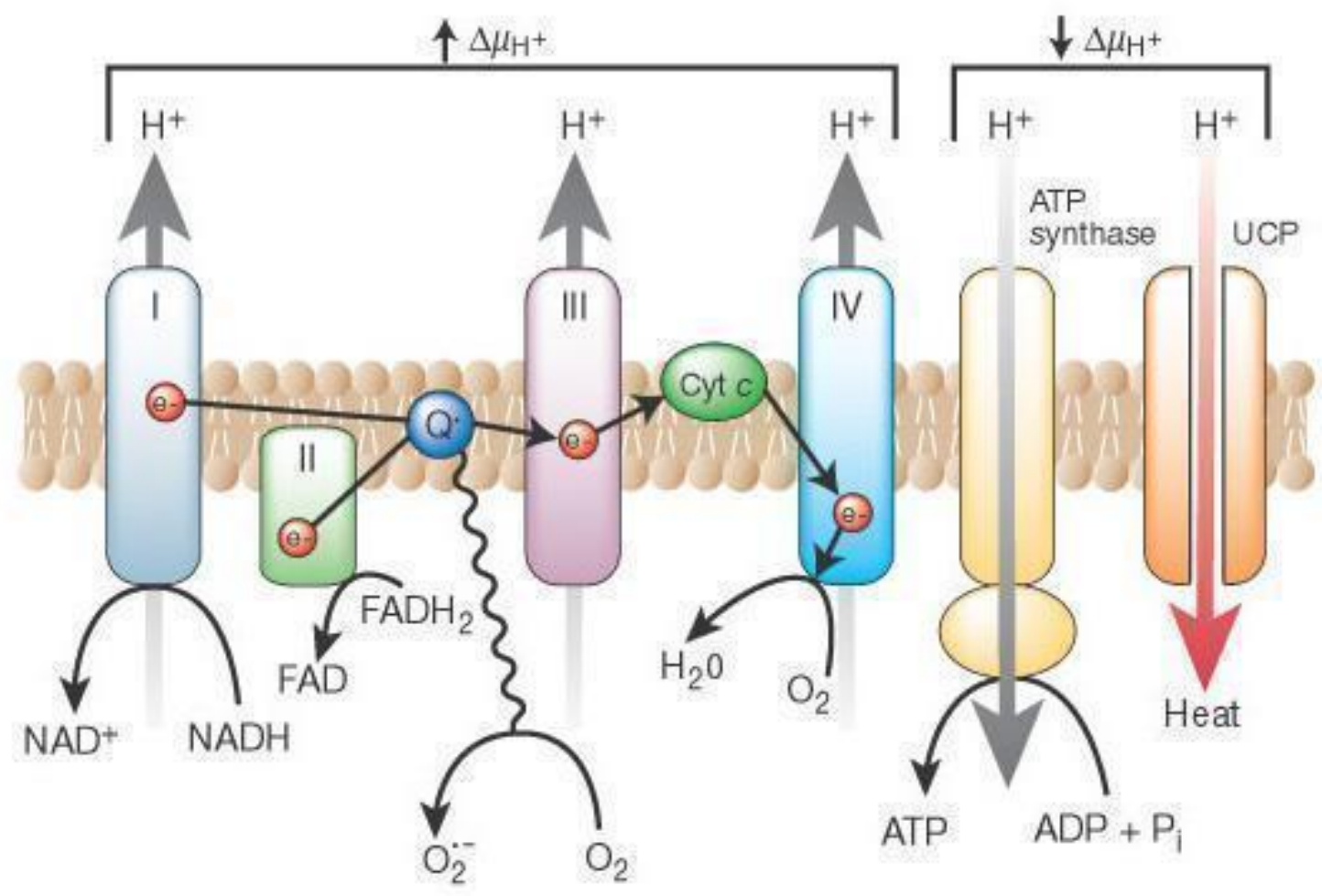

Figure 2. Illustration of the mitochondrial electron transport chain. As electrons as passed down from left to right, several of the complexes pump protons into the intermembrane space which drive ATPase activity and bioenergy production. Occasionally, electrons leak from the chain onto free oxygen molecules in the mitochondrial matrix, creating superoxide, a common form of ROS, in close proximity to complex I.(Brownlee, 2001)

If it is indeed the case that ROS damage DNA, the proximity of mtDNA genomes to the main site of ROS production could provide an explanation for the higher mutation rates observed for mtDNA. Despite the close proximity of ROS buildup within the mitochondrial ETC, recent work fails to implicate oxidative stress in somatic mtDNA 
mutation accumulation in animal models; rather mutation patterns implicate mtDNA polymerase errors are the primary cause of point mutations (Ameur et al., 2011; Itsara et al., 2014; Yu et al., 2013). However, the question of whether ROS is an important contributor to heritable mutation in either the nDNA or mtDNA genomes remains open.

Experimental Evolution with C. elegans

Experimental evolution allows insight into the mechanistic details of evolution $(\mathrm{N}$ Colegrave \& Collins, 2008; Nick Colegrave \& Buckling, 2005; Estes \& Teotonio, 2009). It allows comparison of the fitness or phenotypes of populations that have been evolved under particular selective conditions with those of their ancestors. C. elegans nematodes, which are highly fecund, short lived, possess many genetic and genomic resources, and are small enough to manage in large populations under a controlled environment, is currently the best metazoan model organism for using laboratory experimental evolution to understand the genetic mechanisms of evolution. Replicate populations can be propagated for many generations in controlled environments and stored cryogenically, allowing comparisons between evolved and ancestral states (R. B. R. Azevedo et al., 2002; Davies et al., 1999; Estes et al., 2005, 2004; Estes \& Phillips, 2006; Haag-Liautard et al., 2007; Keightley \& Bataillon, 2000; Vassilieva et al., 2000).

Caenorhabditid nematodes have also emerged as an important and frequently used model for studying mitochondrial electron transport chain (ETC) dysfunction and its associated 
biological consequences. From physiological response to oxidative stress (Joyner-Matos et al., 2013; Kayser et al., 2001; Smith et al., 2014) and spontaneous mutation studies (D. R. Denver, 2000; Dee R Denver et al., 2009, 2012, 2004; Estes et al., 2004), the small size, highly differentiated tissues, transparent cuticle, short lifespan, and high fecundity of nematodes make them valuable for multi-generation evolutionary studies. In addition, all multicellular organisms (including worms) share similar mitochondrial metabolism and ETC function, providing insight beyond a single taxon (Westermann, 2010).

I sought to address whether oxidative stress leads to heritable mutation within the germ line using MA experiments with wildtype and ETC mutant $C$. elegans strains. The ETC mutant strain utilized for this experiment was gas-1 ( $f$ c21), named for its heightened sensitivity to volatile anesthetics (Gentry et al., 2013). The gas-1 (fc21) allele is a single base-pair substitution within a nDNA (X chromosome) gene encoding a central component of ETC complex I. Mutations affecting gas-1 and other complex I components typically lead to elevated ROS levels along with reduced $\Delta \psi \mathrm{M}$ and ATP production (Lenaz, Baracca, et al., 2006; Lenaz, Fato, et al., 2006). Compared to the standard laboratory wildtype strain, N2, gas-1 mutants exhibit altered mitochondrial function, reduced and delayed fecundity, and a "slow living" phenotype. I evaluated the effects of the MA treatment on organismal and organellar health using standard fitness assays and subcellular phenotyping. This information was combined with Illumina genome sequence data to provide a comprehensive view of the effects of ETC damage (and associate oxidative stress) on deleterious mutation accumulation in nDNA and 
mtDNA genomes.

\section{Materials and Methods}

Nematode strains and culture conditions

Two strains were utilized in this study: gas-1 ( $\left.f_{c} 21\right)$ and Bristol N2, the standard wildtype control. The gas-1 mutant, created using standard ethyl methanesulfonate mutagenesis, was obtained from the Caenorhabditis Genetics Center (University of Minnesota). This gas-1 mutant was backcrossed to wildtype $\mathrm{N} 2$ for 10 generations - tracking the gas- 1 (fc21) allele with PCR and Sanger DNA sequencing - to create an isogenic ancestral strain, which was stored cryogenically until experimentation. In addition to the gas-1 and N2 ancestral strains, previously-evolved gas-1 and N2 mutation-accumulation (MA) lines developed from these strains were used for the current study. For gas-1, a set of 40 gas-1 MA lines was derived from the offspring of a single C. elegans gas-1 (fc21) hermaphrodite. The lines were bottlenecked by transferring single larval stage 4 (L4) offspring to fresh plates for an average of 43 generations (Min: 35, Max: 47), after which time they were stored cryogenically in a mixture of glycerol and $\mathrm{S}$ buffer $(129 \mathrm{ml} 0.05 \mathrm{M}$ $\left.\mathrm{K}_{2} \mathrm{HPO}_{4}, 871 \mathrm{ml} 0.05 \mathrm{M} \mathrm{KH}_{2} \mathrm{PO}_{4}, 5.85 \mathrm{~g} \mathrm{NaCl}\right)$ at $-80{ }^{\circ} \mathrm{C}$ (Stiernagle, 2006). All lines were incubated at $20^{\circ} \mathrm{C}$ and offspring were transferred to new plates at 4-day intervals. The MA process minimizes the power of natural selection and allows new mutations to accumulate via genetic drift in the independently evolving MA lines (Vassilieva \& 
Lynch, 1999). The MA lines generated from the wildtype N2 strain were obtained from Charles Baer (University of Florida) who evolved replicate lines of a highly inbred N2 stock in the manner described above for an average of 250 generations (Baer et al., 2005). Specifically, this study utilized a subset of these Baer N2 MA lines (5 lines: 523, 526, 529, 553, and 574) for which genome sequences (Dee R Denver et al., 2009) and phenotypic data were available (Joyner-Matos et al., 2013).

Prior to all experimentation, the appropriate C. elegans strains were thawed onto two Nematode Growth Medium (NGM) Lite Petri plates seeded with Escherichia coli strain OP50-1 cultures previously incubated at $37^{\circ} \mathrm{C}$ for 24 hours. Strains were incubated at 20 ${ }^{\circ} \mathrm{C}$ for 2-3 generations by transferring approximately 10 worms per strain to fresh plates to eliminate maternal effects of freezing and any bacterial contamination. During experimentation, nematodes were cultured at $20^{\circ} \mathrm{C}$ on $15 \mathrm{ml} \mathrm{NGM}$ Lite plates seeded with $E$. coli as a food source. For ATP analysis, worms were age synchronized by standard bleach treatment prior to each assay. This process destroys all individuals including gravid hermaphrodites, leaving behind an age-synchronous population of eggs (Hope, 1999). 
Fitness Assays

Fitness was assessed by measuring daily and lifetime fecundity and total lifespan for all C. elegans lines following standard protocols (Baer et al., 2006). Two sets of assays were conducted; the first included all MA lines and the second was conducted to increase sample size for only the five gas-1 MA lines selected for genome sequencing (below). For logistical reasons, the first set of fitness assays was conducted in four separate blocks. Each block consisted of replicates of the N2 control, the gas-1 ancestral strain, and a selection of MA lines. At least ten individuals from each MA line were assayed in parallel with the N2 and gas- 1 ancestral controls for comparison and to account for any inter-assay variation. On day 4 after thawing, five L4-stage worms were transferred to a NGM Lite plate to generate an approximately age-synchronous culture. On day 10, five gravid hermaphrodites from each culture were placed onto individual NGM Lite plates, allowed to lay eggs, and killed after 5 hours. Resulting offspring were picked to individual plates at the $\mathrm{L} 3 / \mathrm{L} 4$ stage (10 offspring from two N2, 5 offspring from four gas-1 progenitors, and 5 offspring from two gas-1 MA individuals). After the start of reproduction, each worm was transferred to a new plate every 24 hours until no more eggs were laid. Plates were incubated for 24 hours at $20^{\circ} \mathrm{C}$ to allow offspring to hatch; offspring were killed with a drop of sodium azide or via exposure to a $4{ }^{\circ} \mathrm{C}$ environment and subsequently stored at $4{ }^{\circ} \mathrm{C}$. Daily and total reproductive output was determined through direct offspring counts on plates contrast stained with toluidine blue. Lifespan 
was measured in days lived from the egg stage; worms were scored as dead if they failed to respond to gentle prodding of the head with a platinum wire.

\section{ROS and Membrane Potential}

ROS and membrane potential $(\triangle \Psi \mathrm{M})$ were measured for individual nematodes using fluorescence confocal microscopy and mitochondria-targeted dyes following previously established protocols (Hicks et al., 2012). Measuring steady state ROS in live animals using this protocol reflects the rates of both ROS generation and scavenging by antioxidant systems, and is an accurate measurement of both $\triangle \Psi \mathrm{M}$ and oxidative stress in vivo (Smith et al., 2014). Worms were raised on live E. coli at $20{ }^{\circ} \mathrm{C}$. Prior to each assay, age synchronized worms were labeled with specific fluorescent dyes appropriate for each experiment (MitoSOX Red for steady-state ROS levels and MitoTracker Red CMXRos for average $\Delta \Psi \mathrm{M})$. A second age-synchronized set of worms from the same strain and MA line was incubated without dye to serve as the control. After dye exposure, worms were washed and transferred to plates with unlabeled $E$. coli. The worms were allowed to purge any labeled E. coli from their digestive system over a period of one hour. Just before imaging, worms were paralyzed with $5 \mathrm{M}$ levamisole, which prevents depolarization of skeletal muscle within the specimen.

For each set of strains and MA lines, images of the pharyngeal bulb were collected. This structure - used by worms to consume bacteria - was chosen because of its mitochondria- 
rich nature as well as the simplicity to visualize. A high resolution wide field Core DV system (Applied Precision) equipped with an Olympus IX71 inverted microscope mounted with a Nikon Coolsnap ES2 HQ camera (Advanced Light Microscopy Core Facility, Oregon Health and Science University, Portland, OR) was used to capture images. ROS and $\triangle \Psi \mathrm{M}$ levels were obtained by manually encirclingthe pharyngeal bulb within each deconvolution-optimized image and obtaining the intensity of the area using ImageJ software (NIH). ROS and $\triangle \Psi M$ levels were obtained by calculating the difference between average maximum pixel intensities (across z-stacks for each individual) between labeled experimental and unlabeled control worms. Unlike other methods such as whole-worm lysate, this method does not cause disruption and release of organellar and intracellular contents, which can lead to increased ROS via the release of free iron and other such reactions (Hicks et al., 2012).

ATP levels

Standing ATP levels were determined for each strain using a luminescence ATP detection kit (Invitrogen, Carlsbad, CA) and quantified using a TECAN M200 96-well plate reader. Two separate experiments were performed. The first involved four replicate assays of N2, the gas-1 ancestor and the five sequenced gas-1 MA lines. The second involved four replicate assays of N2, the gas-1 ancestor, and the five sequenced N2 MA lines from Denver et al. (2009) (Dee R Denver et al., 2009). Equal numbers of replicates from all strains were included in each assay block. The strains were age synchronized 
using the previously mentioned method and incubated for 2 (N2/N2 MA lines) or 3 (gas1/gas-1 MA lines) days. Young adult worms were collected, washed, and centrifuged three times in M9 buffer, and pellets were placed in $1.7 \mathrm{~mL}$ Eppendorf centrifuge tubes in a total volume of $1 \mathrm{~mL}$. Worms were frozen for 15 minutes at $-80{ }^{\circ} \mathrm{C}$ and thawed to room temperature for three repetitions, and incubated in a $100{ }^{\circ} \mathrm{C}$ water bath for 15 minutes to halt ATPase activity and release ATP into solution. After freeze/thawing, the solution was sonified ( $20 \%$ amplitude, twice for 12 seconds each) and centrifuged at $4{ }^{\circ} \mathrm{C}$ and $\sim 10,000 \mathrm{x}$ g for 12 minutes. $10 \mu \mathrm{L}$ of the supernatant was placed directly into a Corning 96 Flat White plate along with $90 \mu \mathrm{L}$ of Standard Reaction Solution and luminescence readings were obtained at $28^{\circ} \mathrm{C}$ according to the manufacturer's instructions (Invitrogen, Carlsbad, CA). Due to variable fecundity amongst strains and controls, all ATP measurements were normalized by protein content ( $\mathrm{mM}$ of ATP $/ \mu \mathrm{g}$ of protein) via bicinchoninic acid assay (BCA). Corrected averages for standards were calculated (avg. reading of standard - avg. reading of zero standard) to produce a linear equation. Corrected averages of sample readings were calculated in the same fashion, and ATP levels were determined by utilizing the linear equation created. For BCA determination, $25 \mu \mathrm{L}$ of the same supernatant was placed in a Greiner 96 Flat Transparent plate designed for color determination, along with $100 \mu \mathrm{L}$ of the Working Reaction, according to the manufacturer's instructions (Thermo Fisher Scientific, Waltham, MA). The plate was incubated for 30 minutes at $37^{\circ} \mathrm{C}$ and cooled to room temperature. Absorbance was measured at a wavelength of $562 \mathrm{~nm}, 25 \mathrm{flashes} / \mathrm{well}$, at $37^{\circ} \mathrm{C}$. Protein levels were calculated in the same manner as previously mentioned. 


\section{Lactate levels}

Measurements of L-lactate were obtained from population samples of nematodes by Estes lab members using a protocol adapted from (Pierre Blier, pers. comm.) (SenooMatsuda et al., 2001). Age-synched populations of young adult worms were rinsed off of culture plates with M9 Buffer and placed into labeled 15-mL conical tubes. The worms were rinsed multiple times with M9 to remove E. coli from the sample. Worms were centrifuged at 800 revolutions/min for 1 minute, decanted, and resuspended with M9. 750 $\mu \mathrm{L}$ of the worm pellet was transferred to a $2 \mathrm{~mL}$ microtube and centrifuged for 10 minutes at $10,000 \mathrm{G}$ in a refrigerated microcentrifuge at $6{ }^{\circ} \mathrm{C}$. Samples were placed on ice and $750 \mu \mathrm{L}$ pf 1-\% trichloroacetic (TCA) acid was added to achieve a final concentration of 5\% TCA acid. Each sample was homogenized and sonicated in 4 repetitions of 30second pulses with 30 second cooling periods on ice. Sample tubes were centrifuged at $10,000 \mathrm{G}$ for 10 minutes. $1 \mathrm{~mL}$ of the supernatant was placed in a new $2 \mathrm{~mL}$ microtube, and TCA acid was neutralized with $76.5 \mu \mathrm{L}$ of $4 \mathrm{M}$ potassium hydroxide $(\mathrm{KOH})$ and centrifuged again at $10,000 \mathrm{G}$ for 10 minutes. $500 \mu \mathrm{L}$ of the supernatant was placed in a new $2 \mathrm{~mL}$ microtube. This resulting product was used for both lactate assay and total protein determination by the BCA method.

For enzymatic determination of L-lactate, $250 \mu \mathrm{L}$ of buffer (hydrazine sulfate and glycine, $\mathrm{pH}$ 9) was first pipetted to wells of a dark, clear-bottomed 96-well plate. $20 \mu \mathrm{L}$ of water (blank), uninoculated nematode growth medium (additional blank), standard, 
and sample were then pipetted to corresponding wells. Next, $25 \mu \mathrm{L}$ of NAD and $2.5 \mu \mathrm{L}$ of L-LDH were pipetted to each well. The plate was incubated at $25{ }^{\circ} \mathrm{C}$ for 1 hour, and fluorescence was measured at $445 \mathrm{~nm}$ and excitation at $340 \mathrm{~nm}$. Lactate data were obtained by subtracting the blank value from all readings, creating a standard curve, and applying the sample data to said curve. BCA determination followed the instructions provided by the ThermoScientific Pierce BCA Protein Assay Kit in clear plates. Final Llactate values were obtained by normalizing all values by protein concentration, yielding comparable data amongst all strains regardless of sample abundance.

\section{Data Analysis}

I first compared the phenotypes of gas-1 to those of wildtype N2. Due to unequal variances between the two strains for fecundity, lifespan, and ROS (Levene's test), data for these traits were compared using Welch's t-tests. $\triangle \Psi \mathrm{M}, \mathrm{ATP}$, and lactate were compared using standard t-tests $(\alpha=0.05)$. I next compared the gas-1 MA lines to their gas-1 ancestor and wildtype. Tests for fecundity differences among N2, gas-1, and the gas-1 MA lines, nonparametric post-hoc tests were utilized (Wilcoxon rank-sum test). Survival curves were analyzed with the Cox proportional-hazards regression model. I next asked whether there were differences among the three major experimental treatments (N2 wildtype, gas-1 mutant, and evolved gas-1 MA lines) using one-way analyses of variance (ANOVA). The above analyses were performed in JMP9/11 (SAS Statistical Inc., Cary, NC) or IBM SPSS Statistics (IBM Corporation, Armonk, NY). 
ROS data for the five N2 MA lines included in this study were obtained from Joyner Matos et al. (Joyner-Matos et al., 2013).

\section{Results}

Phenotypic variation between N2 and gas-1 ancestral strains and among fitness assays

There was no significant effect of assay block for any life-history trait measured for the N2 control and gas-1 progenitor strains. However, the two strains expressed different patterns of variation across assays for early, late, and total reproduction as evidenced by significant interaction effects in tests of the two-factor ANOVA model: $Y=m+$ strain + block $+($ strain $x$ block $)+e$ using the EMS option in JMP 9.0 (SAS) with strain modeled as a fixed effect and block and the interaction term as random effects.

Fitness assays revealed that the gas-1 progenitor has lower lifetime fecundity (Welch's F $=139.5, \mathrm{P}<0.0001)$ as well as a lower variance in fecundity (Levene's $\mathrm{F}=48.38, \mathrm{P}<$ 0.0001) in comparison to the N2 controls (Fig. 3). In addition, fertilized but unhatched eggs were counted during the fitness assay for all lines. Statistical analyses revealed that the gas-1 progenitor, gas-1 MA lines and N2 unhatched egg counts were significantly different (ANOVA, $\mathrm{F}=6.552, \mathrm{P}<0.0015$ ) such that the gas -1 progenitor and MA lines exhibited higher amounts of unhatched eggs compared to the N2 wildtype, but were not different when compared to each other (Tukey's HSD, $\alpha=0.05$ ). Furthermore, the gas- 1 
progenitor exhibited delayed and slightly extended reproductive schedule along with higher rates of survival (Cox Proportional Hazard $\mathrm{X}^{2}=12.26, \mathrm{P}=0.0005$ ) and lived up to 14 days longer than wildtype (Fig. 3). These data point to a slow-aging phenotype in the gas-1 progenitor. In agreement with previous data on isolated mitochondria (Korshunov, Skulachev, \& Starkov, 1997), fluorescence microscopy indicated that the gas-1 progenitor has higher ROS levels (Welch's $\mathrm{F}=26.03, \mathrm{P}<0.0001$ ) and a greater variance (Levene's $\mathrm{F}=35.80, \mathrm{P}<0.0001$ ) in ROS compared to wildtype levels (Fig. 4). Our findings also show higher $\triangle \Psi \mathrm{M}$ in the gas-1 progenitor than wildtype (Fig. 5). Finally, ATP levels were lower in the gas-1 progenitor than wildtype N2 (t-test, $\mathrm{P}<.05$ ).

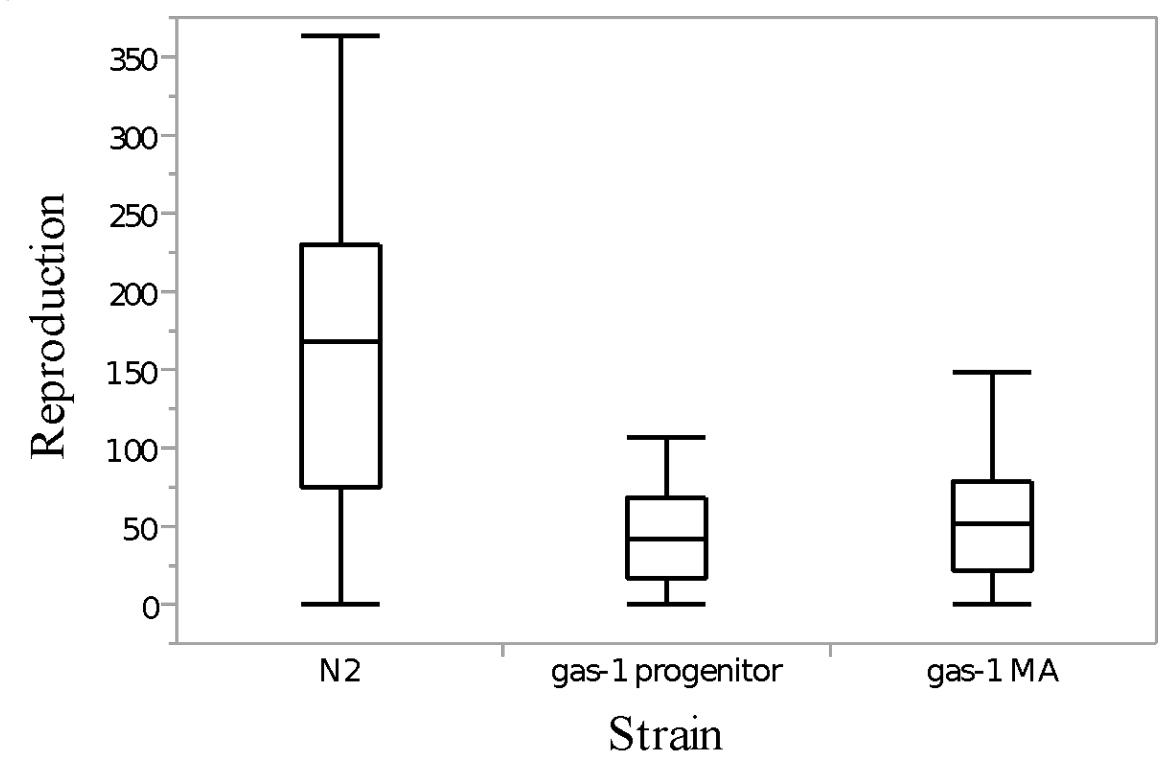

Figure 3. Box and whisker plot depicting fecundity (total individual offspring production) in wildtype N2, gas-1 progenitor, and gas-1 MA lines. Whiskers represent range, center line is the median, and sections are quantiles. gas-1 progenitor has a lower lifetime fecundity $(\mathrm{P}<0.0001)$ and variance $(\mathrm{P}<0.0001)$. MA line fecundity did not decline relative to the gas-1 progenitor. 


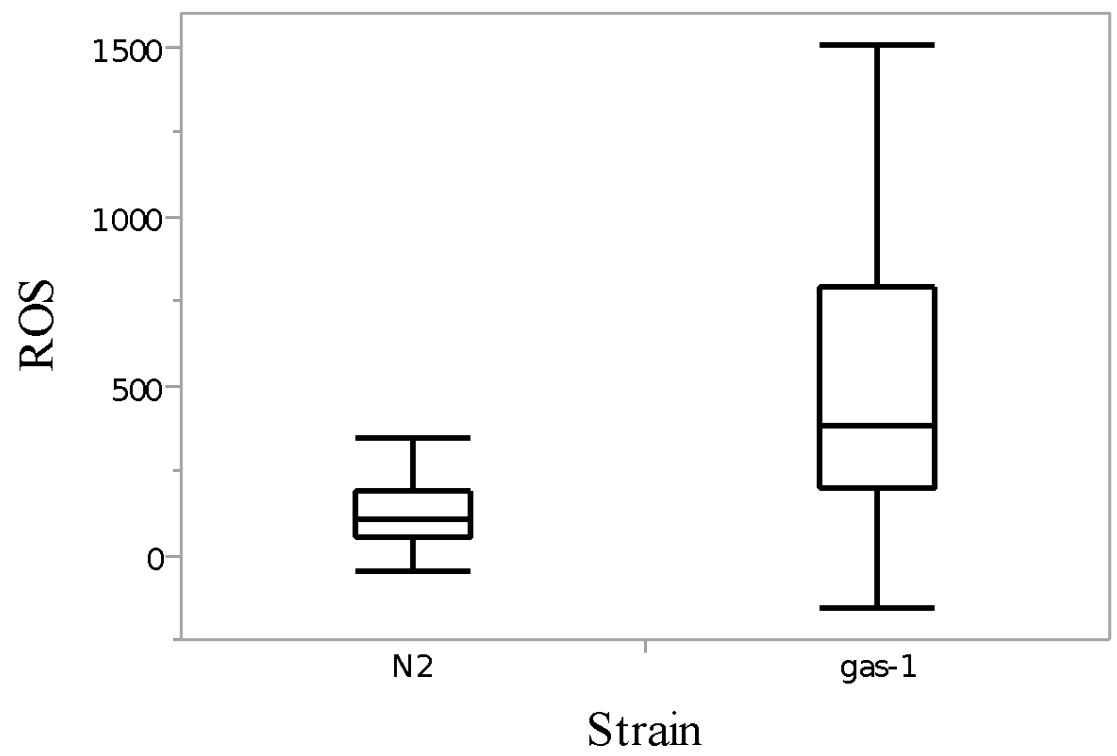

Figure 4. ROS levels of gas-1 progenitor and wildtype N2. gas-1 mutants display higher ROS levels (P < $0.001)$ and greater variance $(\mathrm{P}<0.0001)$.

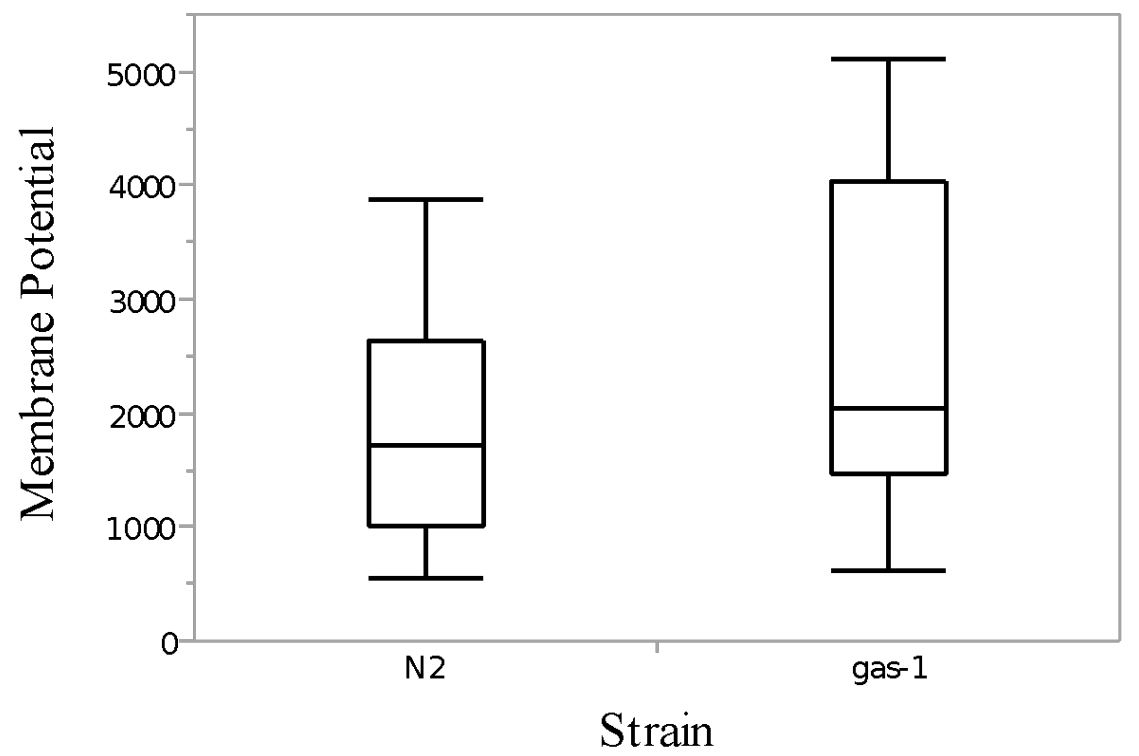

Figure 5. $\Delta \Psi \mathrm{M}$ measurements of gas-1 progenitor and wildtype N2. gas-1 mutants have a higher $\Delta \Psi \mathrm{M}$ compared to wildtype $(\mathrm{P}<0.0001)$. 
Phenotypic variation among N2, gas-1, and gas-1 MA lines

Observations made during the MA experiment suggested that gas-1 MA lines were perhaps suffering higher rates of deleterious mutation accumulation as compared to wildtype N2 MA lines. Specifically, the average frequency with which gas-1 MA lines had to be reconstituted from backup plates was 0.229 per generation; by contrast, a nearly twofold lower frequency of 0.121 per generation was estimated for wildtype N2 MA lines (Dee R Denver et al., 2004). Significant phenotypic variation was observed among gas-1 MA lines for all reproductive traits — but not for lifespan — using tests of nested randomeffects ANOVA model: $Y=m+$ block + MA line(block) $+e$ using the EMS option in JMP 9.0 (SAS), consistent with the buildup of mutational variance in these independently evolved lines.

I next tested for phenotypic differences between the major experimental treatments (N2, gas-1 ancestor, MA) by testing the two-factor random-effects ANOVA model $Y=m+$ treatment + block $+($ treatment $x$ block $)+e$ using the EMS option in JMP 9.0 (SAS). There was a strongly significant effect of treatment on all traits, no effect of assay block for any trait (even though each block contained a different set of MA lines), but significant treatment-block interaction effects for all reproductive traits except for lifespan. For reproductive traits, the pattern of differences among experimental treatments was as follows: N2 > gas-1 ancestor, and gas-1 ancestor $=$ gas-1 MA lines. The opposite pattern was observed for lifespan: N2<gas-1 ancestor, and gas- 1 ancestor $=$ gas $-1 \mathrm{MA}$ 
lines. Further analysis showed that average fecundity of the gas-1 MA lines did not decline relative to the gas-1 ancestor (Wilcoxon post-hoc comparison, $\alpha=0.05$, Fig. 3). Surprisingly, gas-1 MA lines had a higher fecundity on days 3-5 of reproduction in comparison to the gas-1 progenitor (t-tests, $\mathrm{P}<0.001$, Fig. 6). The survival rates of the gas-1 MA lines and progenitor were statistically indistinguishable (Cox Proportional Hazard $\mathrm{X}^{2}=0.878, \mathrm{P}=0.349$, Fig. 7). Average ROS levels of gas-1 MA lines declined to wildtype levels and exhibited a variance close to wildtype, although still statistically higher (Wilcoxon post-hoc comparison, $\alpha=0.05$, Fig. 8). A similar pattern was observed for $\Delta \Psi \mathrm{M}$, where gas-1 MA lines declined to wildtype levels (ANOVA, $\mathrm{F}=1.693, \mathrm{P}=$ 0.158, Fig. 9). Average ATP levels were lower in the gas-1 MA lines compared to N2 wildtype (t-test, $\mathrm{P}<.01$ ), and both gas- 1 and the gas- 1 progenitor combined were also significantly less than the $\mathrm{N} 2$ wildtype (t-test, $\mathrm{P}<.001$, Fig. 10). ATP levels between the gas-1 progenitor and MA lines were not statistically different (t-test, $\mathrm{P}=0.25)$. Finally, the low fecundity of the gas-1 mutant strain presented a major challenge to the accurate measurements of L-lactate. Additional data are forthcoming; however, preliminary results suggest that gas-1 MA lines have similar levels of L-lactate compared to the N2 or gas-1 ancestral strains. 


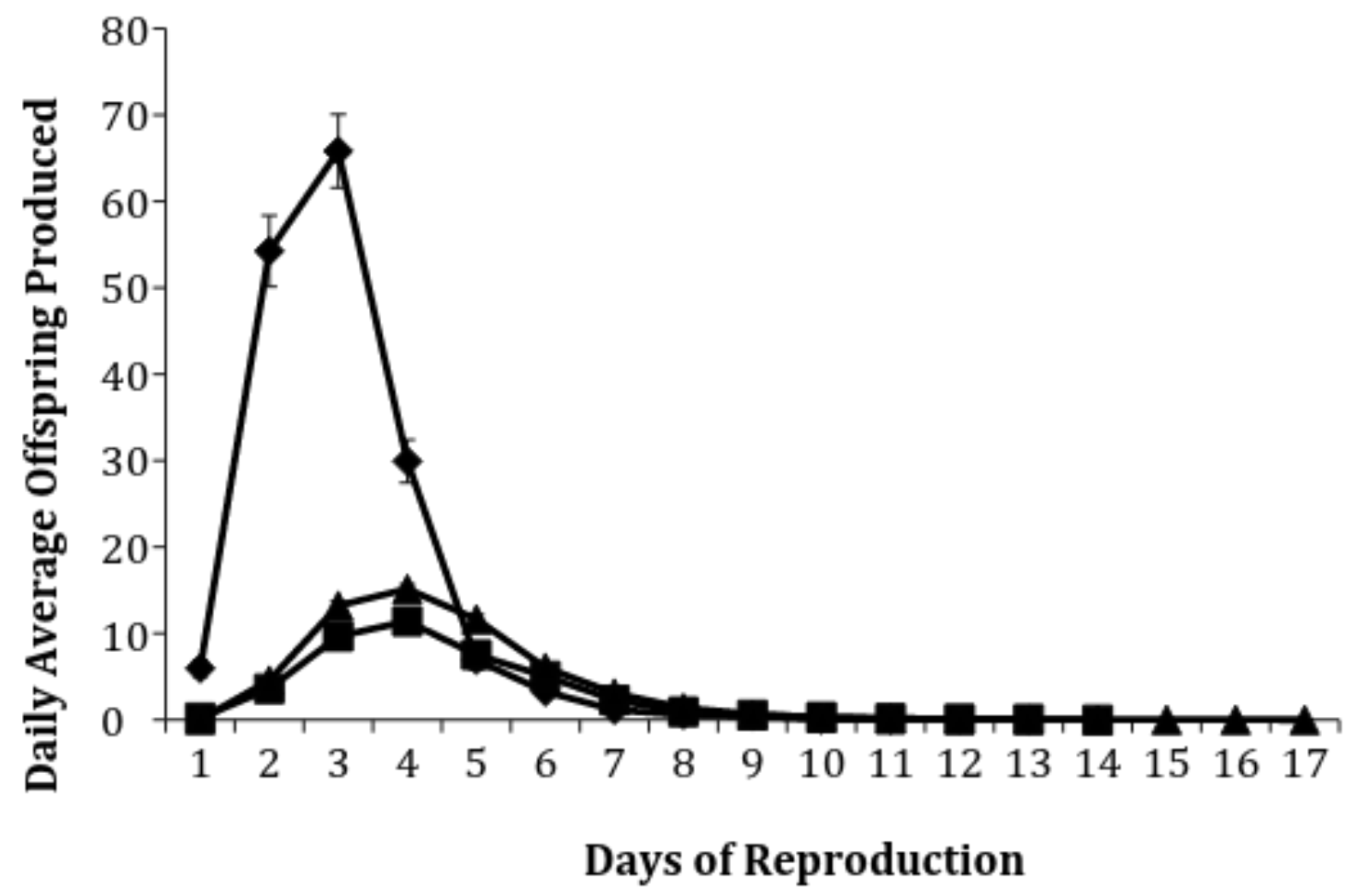

Figure 6. Reproductive schedule on a daily basis of gas-1 MA lines(triangle), gas-1 progenitor(square), and wildtype N2(diamond). gas-1 MA lines exhibited a higher fecundity on days 3-5 compared to the gas-1 progenitor $(\mathrm{P}<0.001)$ 


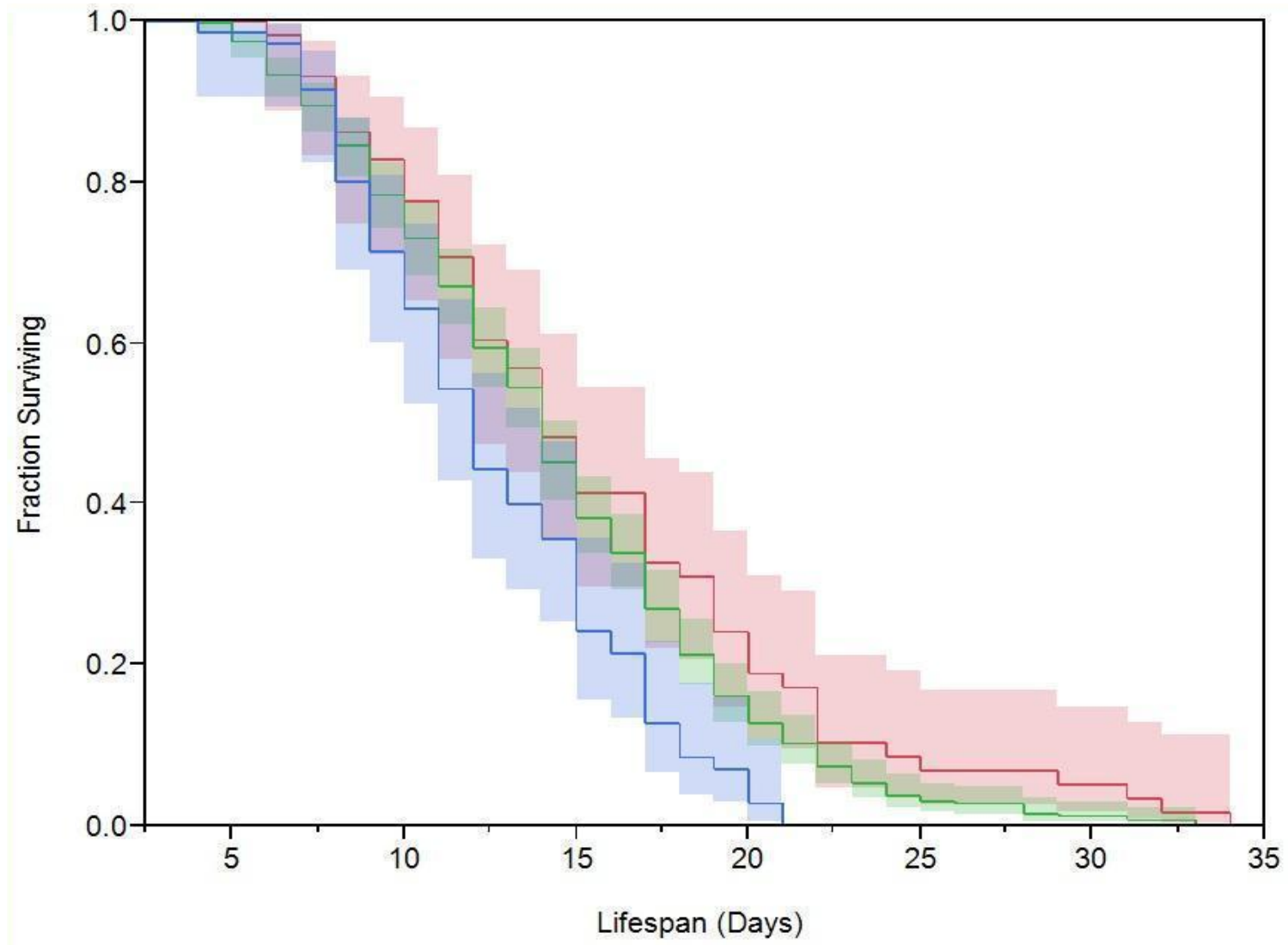

Figure 7. Survival curves of gas-1 MA lines(green, $\mathrm{M}=14.5$ ), gas-1 progenitor(red, $\mathrm{M}=15.8$ ), and wildtype $\mathrm{N} 2$ (blue, $\mathrm{M}=12.0$ ). The gas-1 progenitor has higher rates of survival and can live up to 14 days longer than wildtype $(\mathrm{P}=0.0005)$. MA lines have a similar higher survival and extended maximum lifespan as the gas1 progenitor $(\mathrm{P}=0.349)$. 


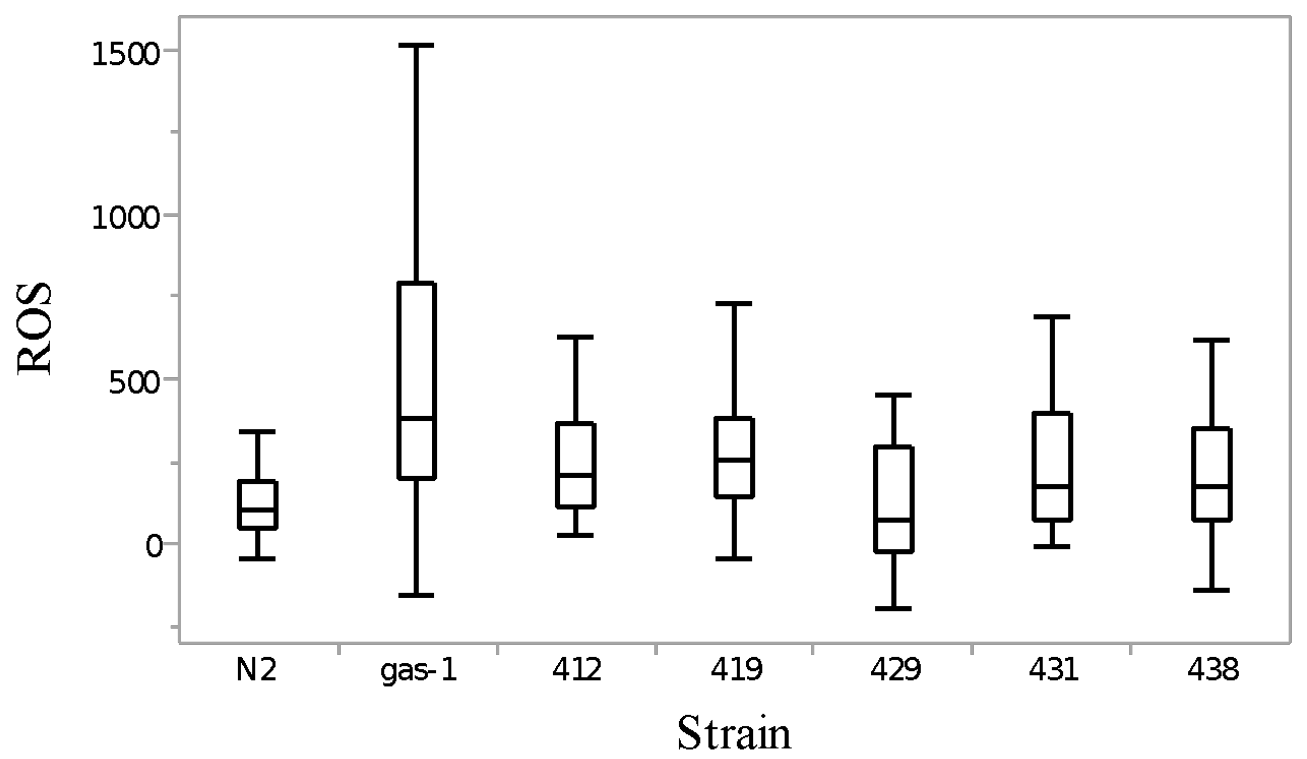

Figure 8. ROS measurements of the same 5 randomly selected gas-1 MA lines, gas-1 progenitor, and wildtype N2. MA lines decline to wildtype levels, and exhibit a variance close to wildtype (although still significantly different $(\mathrm{P}=0.021))$.

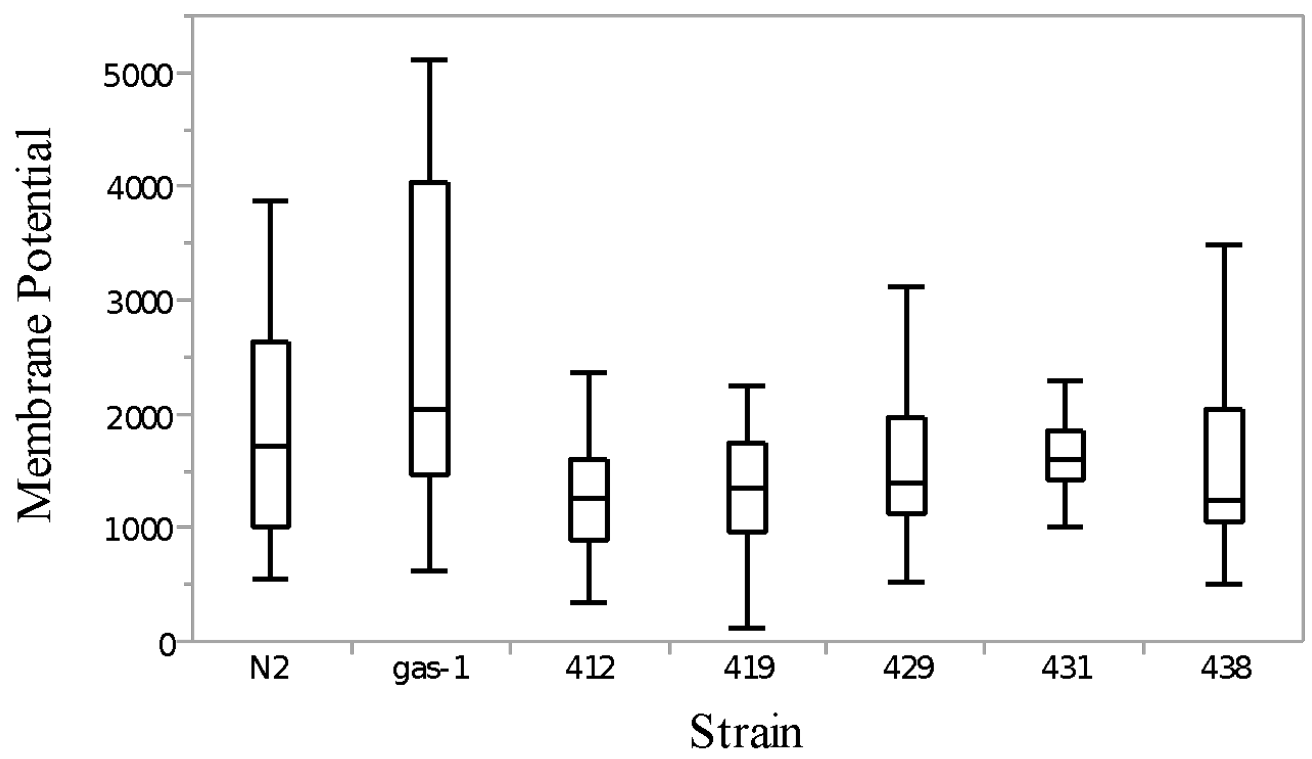

Figure 9. $\triangle \Psi \mathrm{M}$ measurements of the same 5 randomly selected gas-1 MA lines, gas-1 progenitor, and wildtype N2. gas-1 MA lines declined to wildtype levels $(\mathrm{P}=0.158)$. 


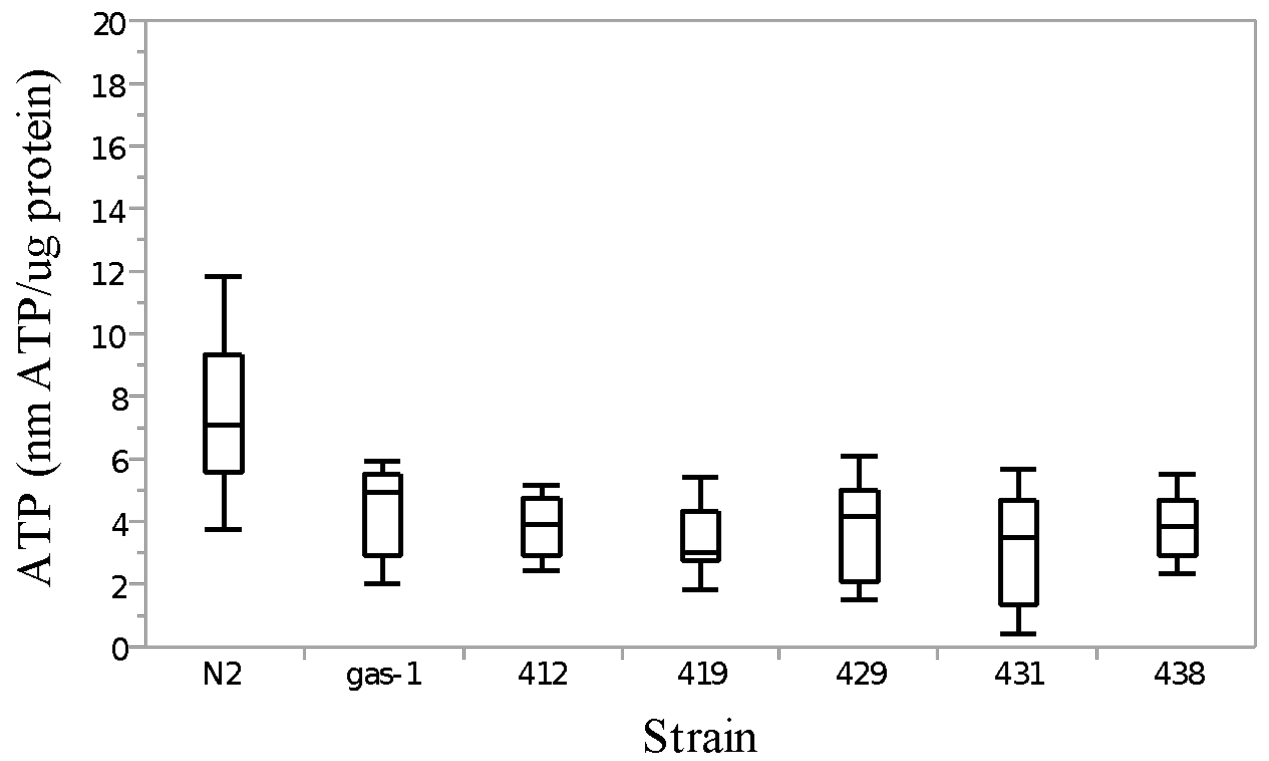

Figure 10. ATP levels of N2 wildtype $(\mathrm{M}=7.47)$, gas-1 progenitor $(\mathrm{M}=4.31)$, and gas-1 MA lines (gas-1 $412(\mathrm{M}=3.90)$, gas-1 $419(\mathrm{M}=3.45)$, gas-1 $429(\mathrm{M}=3.85)$, gas-1 $431(\mathrm{M}=3.10)$, gas $-1438(\mathrm{M}=3.83))$.

Phenotypic variation among N2, gas-1, and N2 MA lines

In addition to measuring phenotypic traits of gas-1 MA lines, select phenotypic assays were conducted on the previously described subset of Baer's N2 MA lines (Baer et al., 2005; Dee R Denver et al., 2009) for comparative purposes (Table 1). I first note that ROS levels were previously assessed for these lines (Joyner-Matos et al., 2013). Average $\triangle \Psi \mathrm{M}$ for the N2 MA lines was significantly indistinguishable from their wildtype N2 progenitor (t-test, $\mathrm{P}=0.19$ ), but significantly lower compared to both the gas -1 progenitor (t-test, $\mathrm{P}<0.0001)$ and gas-1 MA line average (t-test, $\mathrm{P}<0.0001)$. Average ATP level of the N2 MA lines was not significantly different from their N2 wildtype progenitor ( $\mathrm{t}$-test, $\mathrm{P}=0.87$ ), but significantly higher compared to both the gas -1 
progenitor (t-test, $\mathrm{P}<0.001$ ) and gas-1 MA line average (t-test, $\mathrm{P}<0.0001$ ) (Table 1,

Fig. 11).

\begin{tabular}{|c|c|c|c|}
\hline Measurment (units) & $\mathrm{N}$ & Strain & Mean (SE) \\
\hline \multirow[t]{12}{*}{ ATP (nM/ug protein) } & 34 & gas-1 progenitor & $3.311(0.388)$ \\
\hline & 14 & gas-1 412 & $3.899(\mathbf{0 . 6 0 4})$ \\
\hline & 14 & gas-1 419 & $3.453(0.604)$ \\
\hline & 14 & gas-1 429 & $3.848(\mathbf{0 . 6 0 4})$ \\
\hline & 14 & gas-1 431 & $3.100(\mathbf{0 . 6 0 4})$ \\
\hline & 14 & gas-1 438 & $3.827(0.604)$ \\
\hline & 34 & N2 wt & $6.047(\mathbf{0 . 3 8 8})$ \\
\hline & 20 & N2 523 & $3.628(0.506)$ \\
\hline & 20 & N2 526 & $4.099(\mathbf{0 . 5 0 6})$ \\
\hline & 20 & N2 529 & $6.285(\mathbf{0 . 5 0 6})$ \\
\hline & 20 & N2 553 & $9.364(0.506)$ \\
\hline & 20 & N2 574 & $5.891(0.506)$ \\
\hline \multirow[t]{12}{*}{ PP (rate of decline) } & 26 & gas-1 progenitor & $11.51(\mathbf{1 . 5 7 1 )}$ \\
\hline & 19 & gas-1 412 & $5.326(\mathbf{1 . 8 3 8})$ \\
\hline & 19 & gas-1 419 & $11.53(\mathbf{1 . 8 3 8})$ \\
\hline & 20 & gas-1 429 & $9.800(1.792)$ \\
\hline & 21 & gas- 1431 & $5.029(1.748)$ \\
\hline & 19 & gas-1 438 & $11.28(\mathbf{1 . 8 3 8})$ \\
\hline & 29 & N2 wt & $17.12(\mathbf{1 . 4 8 8})$ \\
\hline & 20 & N2 523 & $16.51(\mathbf{1 . 7 9 2 )}$ \\
\hline & 20 & N2 526 & $17.92(\mathbf{1 . 7 9 2})$ \\
\hline & 20 & N2 529 & $13.56(\mathbf{1 . 7 9 2})$ \\
\hline & 20 & N2 553 & $14.49(\mathbf{1 . 7 9 2})$ \\
\hline & 20 & N2 574 & $16.32(\mathbf{1 . 7 9 2})$ \\
\hline \multirow[t]{12}{*}{ ROS (corrected luminescence) } & 37 & gas-1 progenitor & $499.18(41.91)$ \\
\hline & 45 & gas-1 412 & $267.98(\mathbf{3 8 . 0 0})$ \\
\hline & 43 & gas-1 419 & $286.86(\mathbf{3 8 . 8 7})$ \\
\hline & 49 & gas-1 429 & $153.86(\mathbf{3 6 . 4 1 )}$ \\
\hline & 45 & gas-1 431 & $247.82(\mathbf{3 8 . 0 0})$ \\
\hline & 41 & gas-1 438 & $232.35(\mathbf{3 9} .81)$ \\
\hline & 20 & N2 wt & $183.41(\mathbf{5 1 . 3 1})$ \\
\hline & 20 & N2 523 & $471.36(\mathbf{5 1 . 3 1})$ \\
\hline & 20 & N2 526 & $269.19(\mathbf{5 1 . 3 1})$ \\
\hline & 20 & N2 529 & $282.78(\mathbf{5 1 . 3 1})$ \\
\hline & 15 & N2 553 & $261.53(\mathbf{5 9 . 2 5})$ \\
\hline & 20 & N2 574 & $349.45(\mathbf{5 1 . 3 1})$ \\
\hline \multirow[t]{12}{*}{ MP (corrected luminescence) } & 21 & gas-1 progenitor & $3963.18(\mathbf{1 4 8 . 3 3})$ \\
\hline & 17 & gas-1 412 & $1832.07(\mathbf{1 6 4 . 8 6})$ \\
\hline & 22 & gas-1 419 & 1697.08 (144.92) \\
\hline & 23 & gas-1 429 & $1845.93(\mathbf{1 4 1 . 7 4 )}$ \\
\hline & 17 & gas-1 431 & $1963.36(\mathbf{1 6 4 . 8 6})$ \\
\hline & 19 & gas-1 438 & $2222.48(\mathbf{1 5 5 . 9 4})$ \\
\hline & 19 & N2 wt & $-9 e-15(50.47)$ \\
\hline & 20 & N2 523 & 94.55 (49.19) \\
\hline & 19 & N2 526 & $-5.11(50.47)$ \\
\hline & 19 & N2 529 & $659.25(\mathbf{5 0 . 4 7})$ \\
\hline & & N2 553 & - \\
\hline & 20 & N2 574 & $394.09(49.19)$ \\
\hline
\end{tabular}

Table 1. Summary of physiological characteristics across strains. 


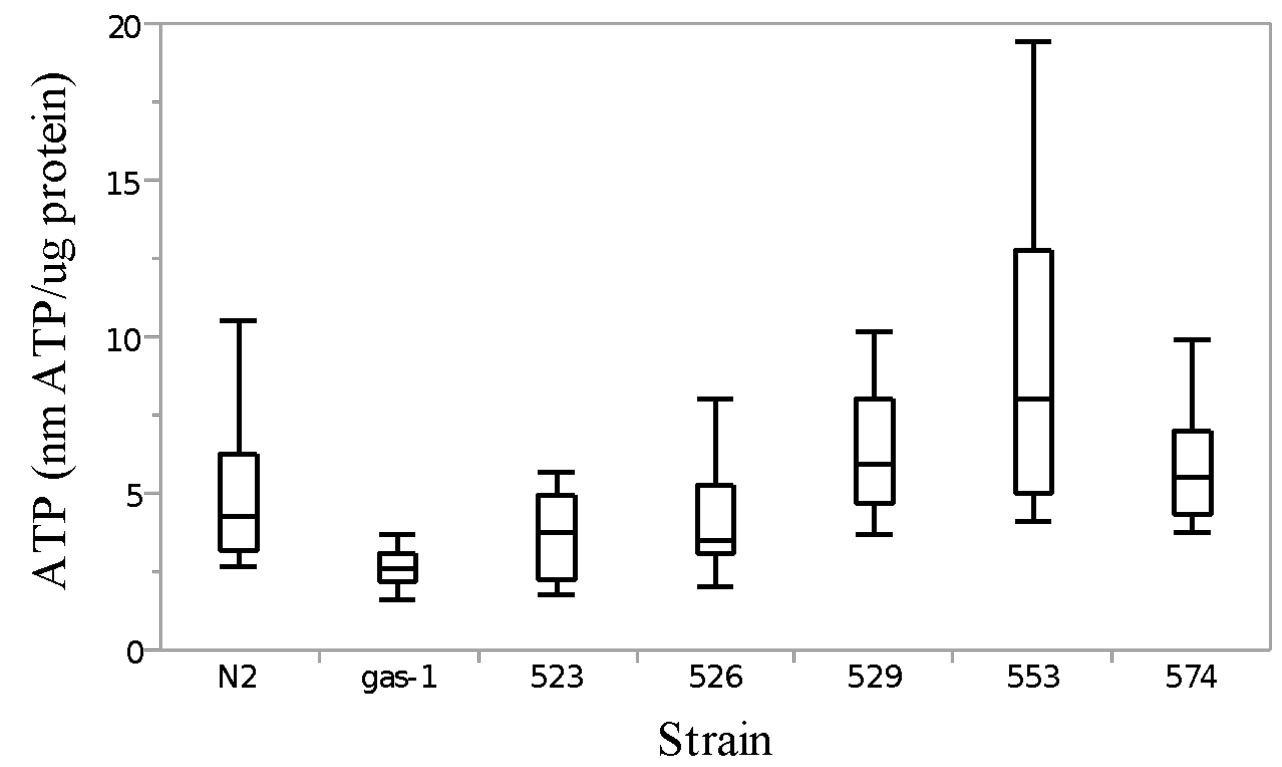

Figure 11. ATP levels of $\mathrm{N} 2$ wildtype $(\mathrm{M}=5.05)$, gas 1 progenitor $(\mathrm{M}=2.61)$, and N2 MA lines (N2 523 $(\mathrm{M}=3.63), \mathrm{N} 2526(\mathrm{M}=4.10), \mathrm{N} 2529(\mathrm{M}=6.28), \mathrm{N} 2553(\mathrm{M}=9.36), \mathrm{N} 2574(\mathrm{M}=5.89))$.

\section{Discussion}

I have applied a variety of techniques to experimentally evolved C. elegans nematodes to analyze the effects of mitochondrial ETC damage on the evolution of fitness and mitochondrial function under mutation accumulation (extreme genetic drift) conditions. I will discuss findings from these experiments in light of additional phenotypic data and Illumina DNA sequence analysis for mutation detection in these lines.

I first assessed daily and total fecundity, survival, and lifespan to characterize fitness of a gas-1 ETC mutant progenitor and MA lines generated from this mutant in comparison to wildtype N2. With the gas-1 progenitor and MA lines, I hypothesized both lower fecundity and lifespan in comparison to controls. In partial agreement with this 
expectation, I discovered that the gas-1 progenitor had lower lifetime fecundity. Beyond this, gas-1 exhibited reduced phenotypic variance for this trait and a slightly extended reproduction period in comparison to wildtype. In contrast to my hypothesis, however, the gas-1 mutant had higher rates of survival, living up to 14 days longer than wildtype. This is in stark contrast to previous reports that gas-1 is a short-lived mutant when incubated at $20^{\circ} \mathrm{C}$ (Morgan, Kayser, \& Sedensky, 2007). The important difference for the current study is that the gas-1 progenitor was extensively backcrossed to N2 wildtype prior to analysis, meaning that all other mutations resulting from EMS treatment (and their accompanying phenotypic effects) were removed. Thus, this study is the first to properly phenotypically characterize the gas- 1 mutation in isolation. Furthermore, we confirmed previous results showing that gas-1 experiences high levels of ROS, and also reported high mitochondrial membrane potential, low ATP levels, and, preliminarily, similar lactate levels for this strain compared to wildtype N2 C. elegans.

\section{Differences in the phenotypic outcome of MA between gas-1 and wildtype lineages}

Surprisingly, neither mean reproductive fitness nor survival of the gas-1 MA lines declined relative to the gas-1 progenitor, and among-line variance in these traits failed to increase (Table 1, Fig. 3, 7). These results differ from those of most other MA studies, including the Baer et al. study that generated the N2 MA lines studied here (Baer et al., 2005) and contrast with the patterns of phenotypic change expected to result from the accumulation of random mutation under drift. Instead, even after $\sim 40$ generations of MA 
treatment, gas-1 MA line fecundity and lifespan remained similar to their progenitor strain. In fact, I observed slightly higher fecundity on days 3-5 of reproduction in these lines compared to the gas-1 progenitor (Fig. 6).

We also assessed the evolution of mitochondrial function in the MA lines generated from gas-1 compared to those generated from the wildtype N2 strain by quantifying ROS, $\triangle \Psi \mathrm{M}$, and ATP levels. Previous work showed that the N2 MA lines had significantly higher in vivo $\mathrm{ROS}$ levels compared to their $\mathrm{N} 2$ ancestor (ANOVA, $\mathrm{F}=4.99, \mathrm{P}=0.0342$ ) (Joyner-Matos et al., 2013). In addition, these MA lines also had marginally higher mean 8-oxodG content than the N2 ancestor (ANOVA, $\mathrm{F}=3.03, \mathrm{P}=0.0965)$. I hypothesized that, owing to the defective ETC complex I (Morgan et al., 2007) and elevated ROS levels of the gas-1 mutant (Fig. 4), MA lines generated from this strain would exhibit even more substantially increased ROS levels than observed for the N2 MA lines; e.g., resulting from additional ETC damage (accompanied by increased ROS generation) accumulated during the MA process. An alternative hypothesis was that ROS levels would decline across generations of MA in these lines if, for example, nematodes reduced their reliance on the ETC for energy metabolism during MA treatment. Observations were consistent with the latter hypothesis: gas-1 MA line ROS levels declined to wildtype N2 levels and had a close to wildtype variance (although still higher) for this trait (Table 1, Fig. 8). For $\triangle \Psi \mathrm{M}$, we found that the gas-1 progenitor had higher levels compared to wildtype $\mathrm{N} 2$, which could result if $\triangle \Psi \mathrm{M}$ is not being dissipated by ATP production in this mutant (Brand, 2000). The low ATP levels observed for gas-1 
compared to N2 (Table 1, Fig. 10, 11) are consistent with this idea. Across generations of MA, average $\triangle \Psi M$ decreased to wildtype levels in the gas-1 lines (Table 1, Fig. 9) while ATP levels remained low compared to N2 and unchanged from that of the gas-1 progenitor (Table 1, Fig. 10). Conversely, $\Delta \Psi \mathrm{M}$ showed a weak tendency to increase across MA generations among the four N2 MA lines measured thus far (Table 1) while ATP levels did not significantly differ between wildtype $(\mathrm{M}=6.05 \mathrm{nM} \mathrm{ATP} / \mu \mathrm{g}$ protein $)$ and N2 MA (M = $5.85 \mathrm{nM} \mathrm{ATP} / \mu \mathrm{g}$ protein) (Table 1, Fig. 11).

To further characterize the gas- 1 strain and understand the potential differences in evolutionary patterns between the gas-1 and N2 MA lines, Griselda Velasco (Estes lab) performed an assay of pharyngeal pumping rates in these lines. Contractions of the pharyngeal bulb are part of typical feeding behavior in C. elegans. The rate of pharyngeal contractions can be easily quantified in age synchronous nematodes and is interpreted as a proxy of chronological age, correlated with many other types of age related decline (Collins, Huang, Hughes, \& Kornfeld, 2007). This trait was also found to be a reliable predictor of the population-level frequency of a large mitochondrial DNA deletion in $C$. briggsae nematodes, with pumping rates being negatively correlated with mtDNA deletion frequency (Estes et al., 2011). At the young adult state, the gas-1 mutant pumped at about half the N2 wildtype rate (see Day 1 in Fig. 12); however, while rates of pumping in the $\mathrm{N} 2$ strain declined with age as expected, gas-1 rates remained more stable. Rates measured for the five selected gas-1 and N2 MA lines did not differ significantly from their respective progenitor strains. Comparison of the two sets of 
strains—gas-1 and gas-1 MA vs. N2 and N2 MA—showed a substantial difference (ttest, $\mathrm{P}<0.0001$ ) such that the gas-1 set had overall lower rates of pharyngeal pumping than the $\mathrm{N} 2$ set. We conclude that both pharyngeal pumping rate and the age-correlated rate of decline in pumping rate is influenced by the presence of the gas-1 mutation, but is not substantially affected by the mutation accumulation process given that MA lines did not differ from substantially from their respective progenitor strains. Together with the finding of low ATP, extended lifespan, and delayed and extended reproductive period in gas-1, results of the pharyngeal pumping assays suggest that this mutant could be characterized as "slow-living". 


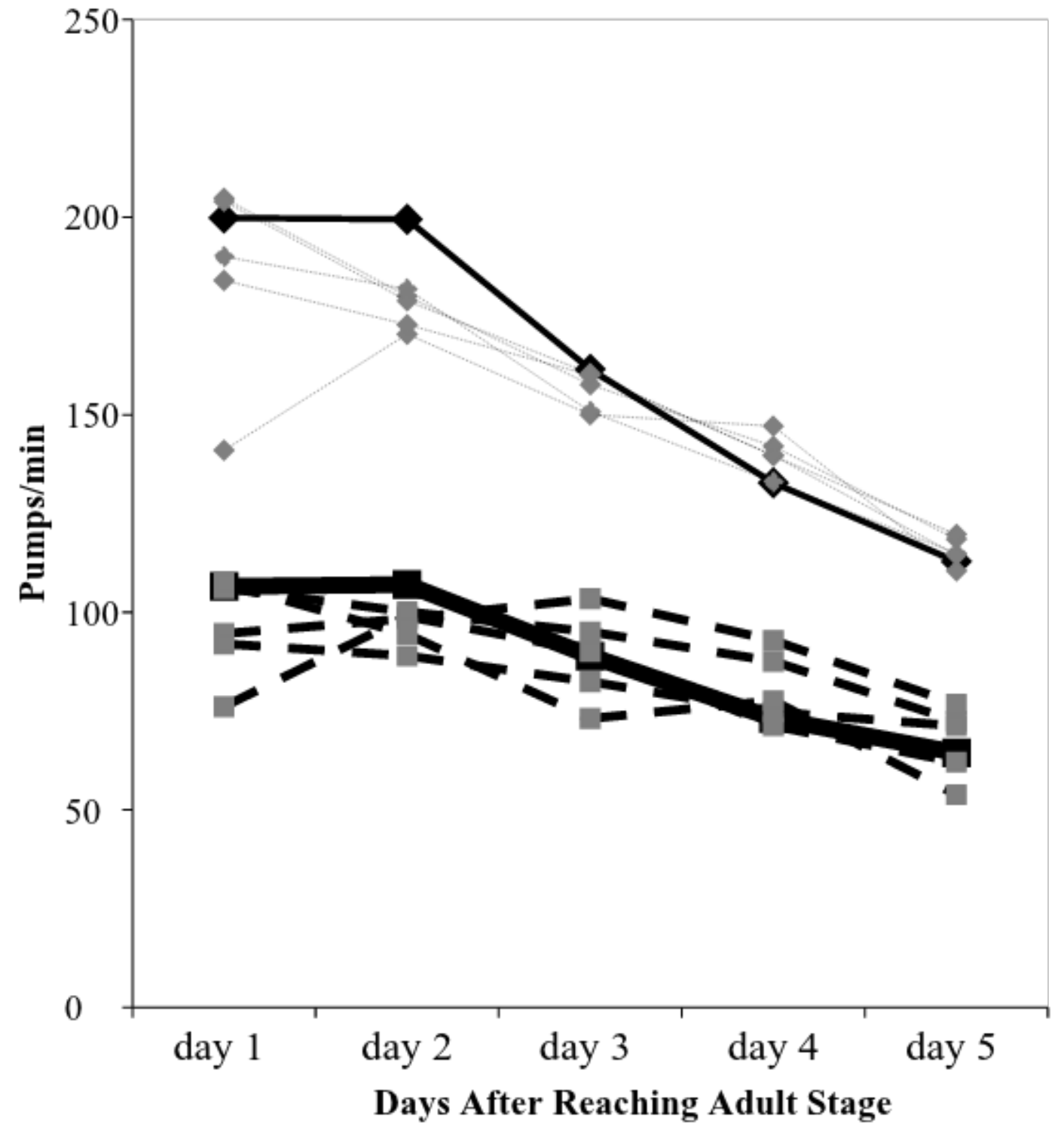

Figure 12. Pharyngeal pumping rates of 5 randomly selected gas-1 (square gray marker, thick dashed lines) and N2 MA lines (diamond gray marker, thin dashed lines), gas-1 progenitor (square black marker, thick solid line), and wildtype N2 (diamond black marker, thin solid line). gas-1 progenitor and gas-1 MA lines did not exhibit different pumping rates or exhibit an age-related decline in comparison to wildtype. 
Relationship of gas-1 phenotypes to transcriptional profiles

The gas-1 mutant phenotypes reported above make sense in light of previous work showing that, while this mutant exhibits high levels of ROS and elevated oxidative stress, it also upregulates expression of numerous cellular metabolic pathways (i.e., OXPHOS, pyruvate metabolism, glycolysis, the TCA cycle, and pathways of amino acid, carbohydrate, fatty acid, and ketone metabolism), and cellular defense pathways (e.g., metabolism of cytochrome P450 and glutathione) (Falk et al., 2008). The latter is consistent with the fact that DAF-16, the single FOXO transcription factor present in $C$. elegans, is constitutively translocated to nuclei in this strain; this event normally occurs only under conditions of starvation, oxidative and other forms of stress in wildtype nematodes (Kondo et al., 2005). daf-16 is negatively regulated by an insulin/insulin-like growth factor (IGF) signaling pathway and, in response to environmental stimuli, regulates approximately 100 genes encoding cell protecting products such as heat shock proteins and antioxidants $(\mathrm{Hu}, 2007)$. The presence of DAF-16 in the nuclei of gas-1 mutants could result from either or both its high intracellular ROS levels or reduced rates of pharyngeal pumping (if the latter leads to dietary restriction), and therefore account for its extended lifespan and slow-aging phenotype. The former scenario seems more likely since gas-1 worms do not have higher than normal frequencies of dauer larva, which might be expected under diet restriction (pers. obs.). 
Opportunity for physiological (non-genetic) compensation in gas-1 MA lines

The findings reviewed above, including the failure of gas-1 MA lines to decline in fitness and their return to wildtype levels of ROS, are consistent with the action of a physiological compensating mechanism (i.e., one that does not result from accumulated genetic variation in these lines), allowing them to maintain their initially low levels of fitness and avoid extinction. In other words, the transcriptional stress responses already activated in the gas-1 mutant ancestor (above) may have been further induced in the MA lines as a result of accumulated mutational damage and/or physiological stress in these lines. A non-mutually exclusive alternative is that depressed or shifted metabolism served to lower production of harmful ROS in gas-1 MA lines. For instance, even when oxygen is plentiful, cells can shift from use of mitochondrial respiration toward increased reliance on glucose fermentation - a phenomenon known to occur in certain cancers (the Warburg effect;(Warburg, 1956)). Although such "aerobic glycolysis" is far less efficient than aerobic metabolism, the negative consequences of reduced ATP production may be outweighed by the benefits of reduced mitochondrial ROS production. These benefits appear to include prevention of apoptosis (Ruckenstuhl et al., 2009) and increased mtDNA genomic stability (Ericson et al., 2012). The occurrence of such a metabolic shift would be consistent with the observations of low ROS and ATP in the gas-1 MA lines, and would also predict higher levels of L-lactate - the major end product of glycolysis in C. elegans. However, our preliminary results showing similar lactate levels in the gas-1 
mutant progenitor compared to all other lines are inconsistent with this idea, but answers await additional data for the gas-1 MA lines.

Opportunity for genetic compensation in gas-1 MA lines

The findings of this study are also consistent with the idea that striking differences in evolutionary process may have existed between these MA lines and those generated from a wildtype $\mathrm{N} 2$ genetic background. Apart from the presence of the gas-1 mutation, another key difference between the two experiments is that they were initiated from ancestral strains with dramatically different fitness levels. Because the gas-1 mutant had such low starting fitness, this may have increased the likelihood that mutations arising during MA would exert beneficial or compensatory effects while simultaneously increasing the strength of selection for such mutations. Even though nematode populations were maintained in effective population sizes of one, where the role of selection should have been minimized, this treatment would not preclude the opportunity for either embryonic or gamete-level selection. We therefore speculate that gas-1 lines experienced some amount of compensatory or generally adaptive evolution during the MA treatment that allowed them to maintain low fitness and avoid extinction. 
Genome sequencing results support genetic compensation hypothesis and implicate insulin/TOR pathways

In addition to our phenotypic assays, collaborators (D. R. Denver lab, Oregon State University) utilized high-throughput Illumina sequencing to detect single-base pair and indel mutations that arose in the mtDNA and nDNA genomes of the five selected gas- 1 lines during the MA process. These data already existed for the five select N2 MA lines (Dee R Denver et al., 2009). Additionally, because this technique gave approximately 1000-fold coverage for the mtDNA genome (excepting the AT-rich control region where coverage was low and variable), these data were also analyzed to provide information on mtDNA copy number and incidence of heteroplasmies in both the gas-1 and N2 MA lines. mtDNA copy number was discovered to increased 2-4-fold in the generation-40 gas-1 MA lines compared to their mutant ancestor whose mtDNA copy number was the same as wildtype (Fig. 13; R. Wernik, unpubl. data). Interestingly, a similar result was observed for the N2 MA lines; this is consistent with increased mtDNA copy number occurring as a passive consequence of the MA process - perhaps signaling dysregulation of mitochondrial-nuclear genome cooperation - rather than resulting from the presence of the gas-1 allele. Furthermore, gas-1-derived MA lines showed only a weak tendency to harbor increased frequencies of heteroplasmic variants, although they had a slightly higher incidence of single-base substitution type mitochondrial heteroplasmies whereas N2-derived lines higher incidence of indel-type variants. Similarly, the per-generation rate of fixed (i.e., homoplasmic) mtDNA variants was found to be exactly the same in the 
gas-1- and N2-derived MA lines, providing no evidence for elevated mtDNA mutation rates in gas-1 lines - unsurprising given their wildtype levels of ROS (R. Wernik, unpubl. data).

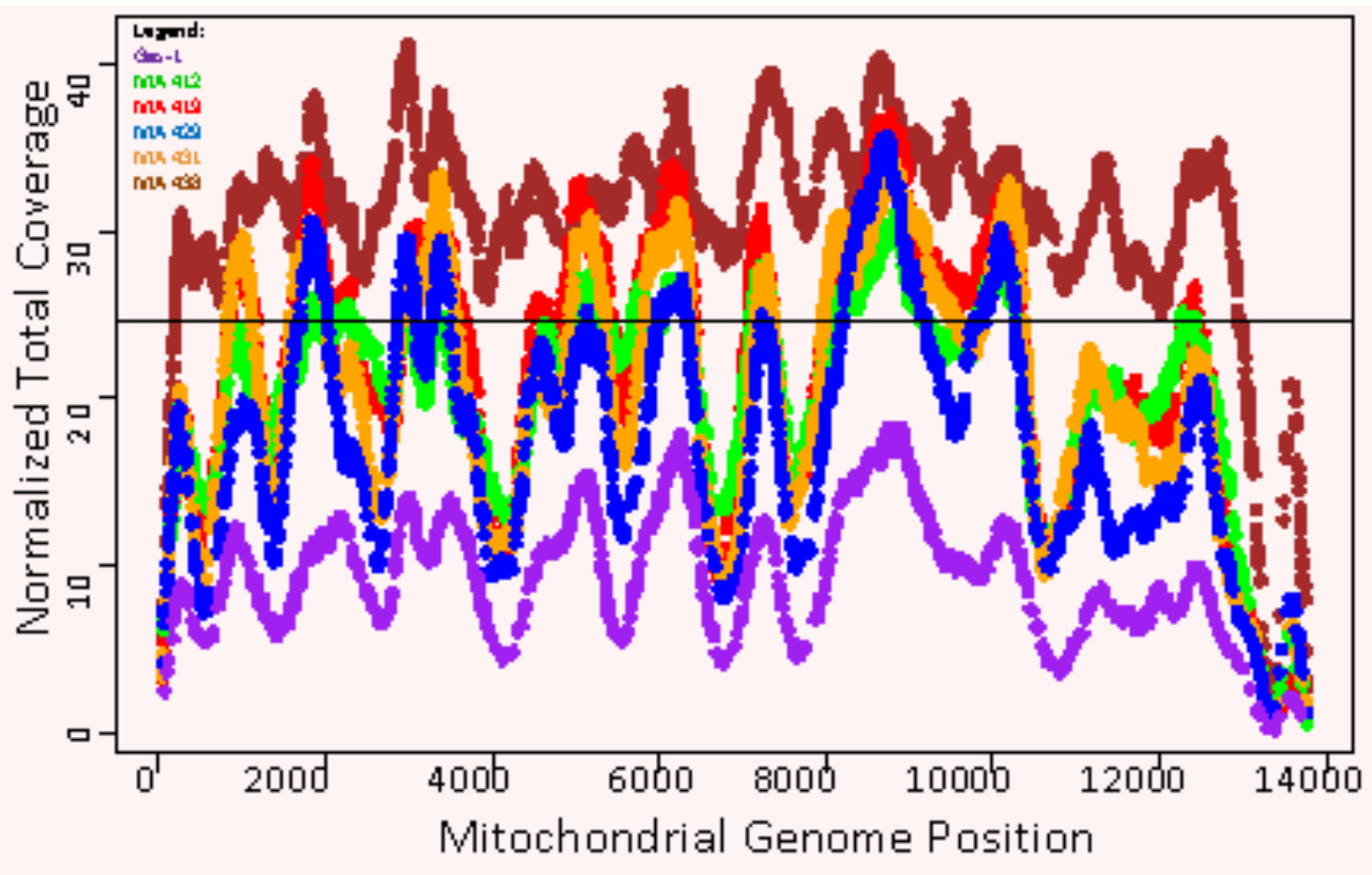

Figure 13. Illumina sequencing results of mtDNA coverage across the mitochondrial genome in gas- 1 progenitor and MA lines.

Like the mtDNA sequence results, analysis of nuclear genome changes showed no difference in the rates of heritable mutations between gas-1- and N2-derived lineages; however, strong evidence emerged that different types of mutations had accumulated in the two sets of lines. First, the ratio of transition mutations to transversions was 1.15 in the gas-1 MA lines compared to 0.45 in the N2 MA lines, indicating a transversion bias for gas-1 lineages. The gas-1 lines also showed a weak tendency to harbor fewer indels 
as compared to $\mathrm{N} 2$ lines. Second, $30 \%$ of mutations acquired by gas-1 lines were in exonic gene regions compared to the $20 \%$ observed for N2 derived lines. Third, of these mutations affecting protein coding regions, the ratio of nonsynonymous to synonymous changes was elevated at 3.33 in gas- 1 lines compared to 2.33 in N2 MA lines. Finally, nDNA mutations identified in gas- 1 lines showed a striking tendency to occur in regions with known or predicted effects on energy metabolism or mitochondrial function; 10 of the 47 of mutation, or $21 \%$, were discovered in such regions. For instance, one of the gas1 MA line acquired a replacement change $(\mathrm{W} \rightarrow \mathrm{C})$ within the alh-2 gene, encoding a mitochondrial aldehyde dehydrogenase. Furthermore, two MA lines independently acquired (different) mutations in the same sequence - F41E7.2 (orthologous to a human sodium/hydrogen exchanger) - providing evidence of convergent sequence evolution. Under a scenario of evolution under pure genetic drift, no such pattern is expected, and indeed, is not observed in the N2 MA lines. More in depth statistical analyses are underway, but we speculate that the non-random pattern of sequence change in the gas-1 MA lines result from compensatory evolution - positive selection for mutations that compensate the deleterious effects of the gas-1 mutation. Preliminary analyses using GeneOrienteer (http://www.geneorienteer.org/) and String (http://string-db.org/) to predict genetic interactions (c.f., (Dee R Denver et al., 2010)) identify $7 / 47$ or $15 \%$ of the mutations in gas-1 MA lines as interacting with the gas-1 genetic network, consistent with selection for sequences changes that compensate for the gas-1 mutation's presumed disruption of its network. 
Intriguingly, $5 / 47$ or $10.6 \%$ of the single-nucleotide mutations identified across gas -1 MA lines occurred within known members of the mammalian target of rapamycin (mTOR) pathway; another $9 / 47$ or $19 \%$ occurred in gene sequences predicted to interact with a member of this pathway. For instance, one of the five sequenced gas-1 MA lines acquired a total of 9 mutations over the course of the experiment; these included substitutions within introns of daf-2, insulin-like growth factor 1 (IGF-1) receptor, and rheb-1, a GTPase involved in the mitochondrial unfolded protein response. (A discussion of daf-2 and the likely effects of the intronic mutation is provided below.) Both genes are central components of the insulin-like signaling pathway and intimately connected with the mTOR pathway (Fig. 14). The mTOR signaling pathway consists of intracellular and extracellular signals that directly regulate cell metabolism, growth, proliferation, and survival (Laplante \& Sabatini, 2009). In healthy eukaryotes, mitochondrial homeostasis is achieved by mechanisms that adapt the number of mitochondria which required for energy production as well as for the supply of metabolic intermediates necessary for cell growth, while removing malfunctioning mitochondria when necessary. In the cytoplasm, a protein complex named mTORC1 couples growth-promoting signals with anabolic processes, in which mitochondria have a vital role. A recent study using C. elegans finds that regulatory processes downstream of mTORC1 affect the rate of mitophagy and regulation of particular transcription factors related to mitochondrial function (Groenewoud \& Zwartkruis, 2013). They also note how mitochondrial function feeds back on mTORC1 via ROS signaling to take part in adapting metabolic processes. In addition to other observations from this study (i.e., increased mtDNA copy number), 
these data suggest that the mTOR pathway may have a critical role in the compensatory effects observed within this study.

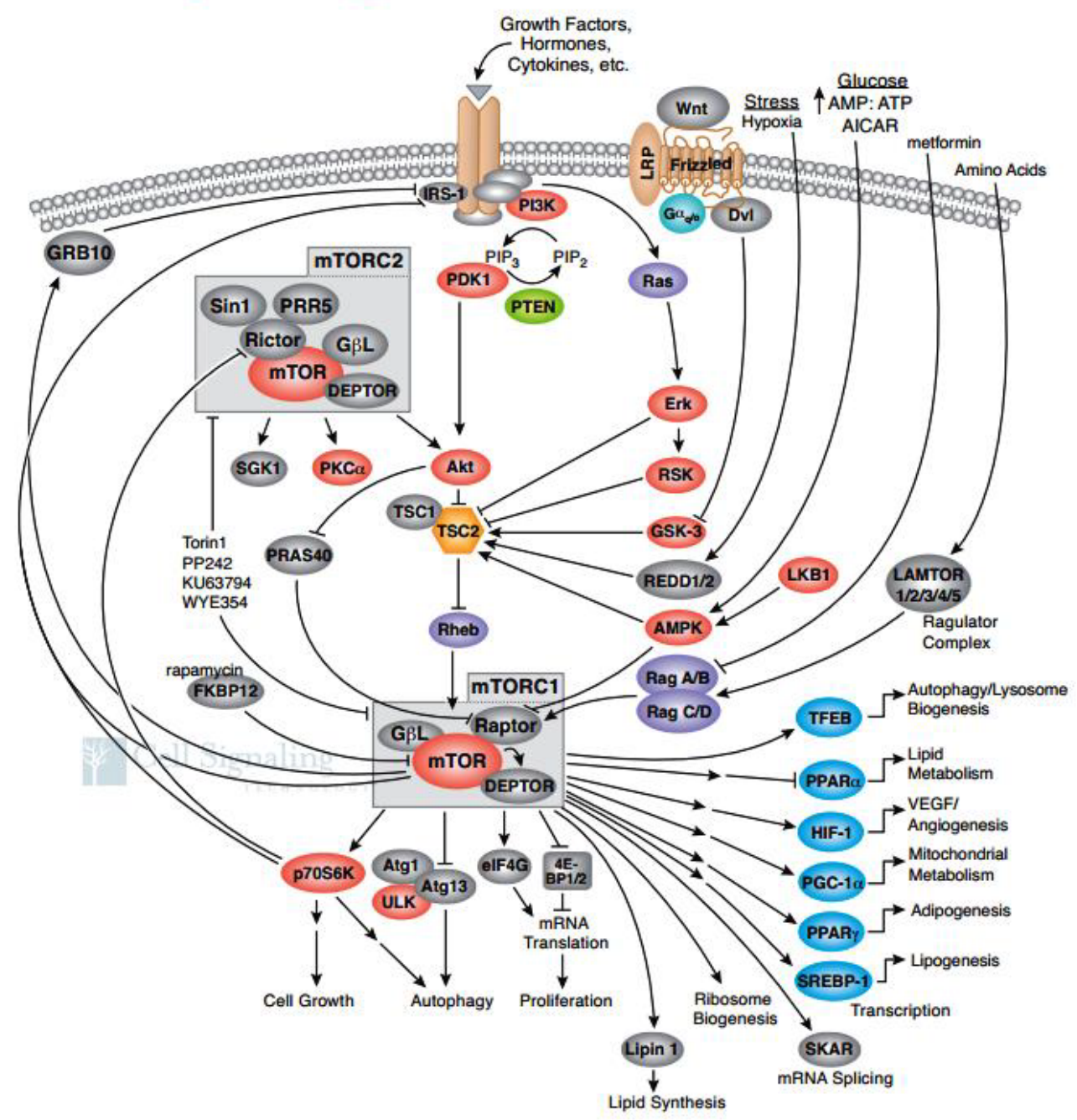

Figure 14. Illustration of the mTOR signaling pathway. (www.cellsignal.com)

DAF-2, the sole IGF-1 receptor in C. elegans, is the best-studied gene in this system owing its central role in regulating longevity and aging, and the finding that mutations of this gene can double nematode lifespan (Kenyon, 2011). It controls expression of genes 
involved in diverse processes such as reproductive development and diapause (i.e., formation of dauer larvae) and resistance to oxidative and thermal stress, hypoxia, and pathogens (Samuelson, Carr, \& Ruvkun, 2007) (Evans, Chen, \& Tan, 2008). The daf2 locus encodes two alternatively spliced transcripts; the resulting isoforms exhibit tissuespecific expression in C. elegans and the abundance of at least one of these appears to be modulated by nutritional status, but their specific functions are unknown (Murphy \& $\mathrm{Hu}$, 2013). Preliminary homology modeling based on human IGF-1 suggests that the intronic daf-2 mutation discovered in the gas-1 MA line from this study occurs near a site of alternative splicing and could thus significantly affect function (D. Bulger, pers. comm.).

A genome-wide RNAi screening of daf-2 mutants found $\sim 200$ genes that were necessary for the mutants' extended lifespan (Samuelson et al., 2007). The researchers measured molecular and behavioral markers indicative of normal aging and extended lifespan to distinguish the difference between accelerated aging and general sickness. They found that endosomal sorting complexes required for transport (ESCRT), involved in vesicle sorting, cellular abscission, and viral budding, to be a major cause of accelerated aging when disrupted. Interestingly, two of the gas-1 MA lines acquired amino acid replacing mutations in different genes involved in endosomal trafficking. The aforementioned MA line containing the intronic mutation in $d a f-2$ was one of these; this line acquiring a mutation in the sel-2 gene, encoding a protein homologous to mammalian neurobeachin that functions to regulate endosomal trafficking during C. elegans development. Another line acquired a mutation in sequence ZC8.6, with homology to human 
phosphatidylinositol 4-kinase type 2-alpha (PI4K), centrally involved in many biological processes including endosomal trafficking (Craige, Salazar, \& Faundez, 2008). This result suggests the possibility that selection on endosomal sorting may have contributed to compensatory evolution in the gas-1 MA lines.

\section{Conclusions}

The combined results reported in this thesis lead to three major conclusions. First, I can neither refute nor support the hypothesis that ROS generates heritable mutation. This is because, although mutation rates were equal to wildtype in the gas-1 MA lines, ROS levels evolved downward in these lines - perhaps due to genetic or physiological compensating mechanisms. Second, the highly non-random pattern of mutation discovered in the nuclear genomes of these lines suggests that selection (i.e., gametic or embryonic selection) can be a powerful force even at population sizes of one.

Third, the results of this study suggest the hypothesis that both physiological and genetic compensation was occurring in the gas-1 MA lines, and that both types of compensation operated via similar or overlapping pathways. 


\section{CHAPTER THREE}

\section{General Conclusions}

This study advances our understanding of mutation and its impact on genotypic and phenotypic variation. First, my results added to knowledge of gas-1 mutant phenotypes, such as delayed and extended reproductive periods, and higher survival rates. Second, my findings support the idea that, even under conditions of strong genetic drift (i.e., evolution in population sizes of one) the gas-1 MA lines acquired compensatory mutations that allowed them to maintain their low levels of fitness and avoid extinction. After undergoing laboratory MA for an average of 43 generations, the gas- 1 MA lines failed to exhibit the expected decline in fecundity compared to the gas-1 progenitor. This coincided with ROS and $\triangle \Psi \mathrm{M}$ declining to wildtype levels in these lines.

Previous work shows that the gas-1 mutant has high levels of ROS as well as upregulated stress response pathways (i.e. heat shock, oxidative, hypoxic, and osmotic stress) (Fig. 15, (Rodriguez, Snoek, De Bono, \& Kammenga, 2013). This may be due to the nuclear localization of $d a f-16$ which could cause the observed extended lifespan and slow aging phenotype where somatic tissue maintenance is prioritized over reproductive capability (Kondo et al., 2005). If this is true within the context of the gas-1 strain, we would predict that that these patterns would be magnified in the MA lines where more mutations 
would lead to increased physiological stress and thus further stress response gene upregulation.

Second, the compensation may be genetic/mutational in nature. Several of the mutations we discovered (e.g. daf-2) seem to occur in the ILS pathway, mTOR, and/or mitochondrial function pathways. This suggests selection for compensatory mutations that may have occurred during the development of the MA lines, and that these mutations were located in pathways that were already being differentially expressed in the gas-1 ancestor. An alternative idea is that mTOR pathway genes became mutational hotspots in the gas -1 background, although it is difficult to imagine the proximal mechanism that would lead to such a pattern. 


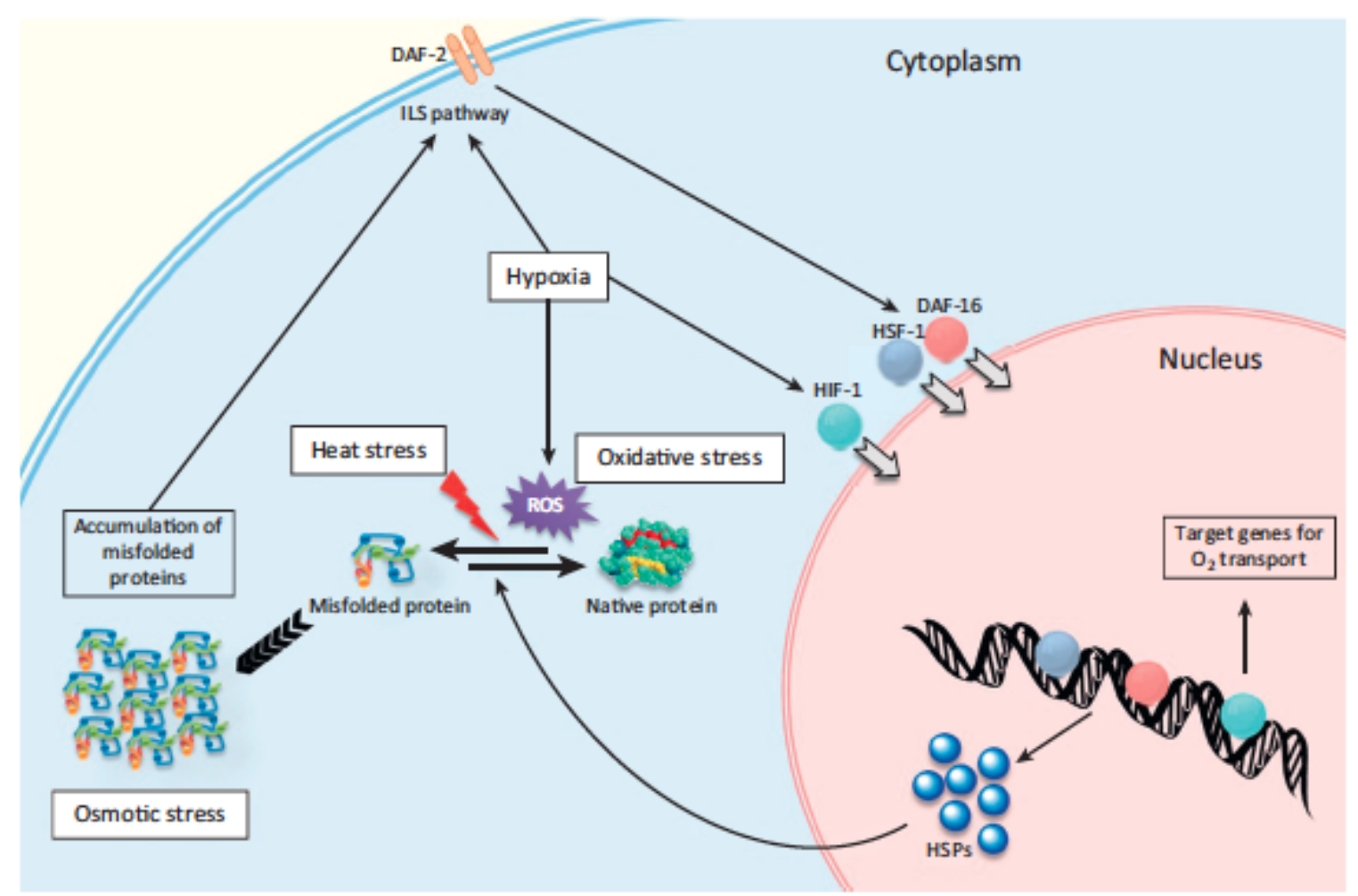

Figure 15. Illustration of the stress response in a C. elegans cell. The stress response mechanism is activated mainly by the ILS pathway (e.g. heat-shock proteins (HSPs) produced as a result of heat stress).

\section{Future directions}

An obvious next step for this research will be to test the individual effects of the putative adaptive or compensatory mutations that arose in the gas-1 MA lines. Our collaborators attempted to backcross one of the gas-1 MA lines to the gas-1 progenitor in order to isolate candidate compensatory mutations that arose in genes near each other on Chromosome III - daf-2, rheb-1, and sel-1 - on a mutant background. Unfortunately, attempts were unsuccessful and — as an alternative — they successfully backcrossed this gas-1 MA line to the wildtype N2 strain; this will be followed by crosses to obtain each 
mutant allele in isolation on the gas-1 ancestral mutant background. Interestingly, they never observed the $r h e b-1$ gene by itself in offspring from these crosses, potentially meaning that it may be deleterious on a wildtype background. If this mutation is beneficial only in the context of the gas-1 mutation, it would conform to the strict definition of a compensatory mutation.

Finally, it is possible that mitochondrial biogenesis or other aspects of organelle dynamics play a role in maintaining fitness of the gas-1 MA lines. Between the L1 and young adult stages, preliminary studies found a larger increase in mtDNA/nDNA in the gas-1 mutant (8.33 to 23.3 ) than in $\mathrm{N} 2$, which may reflect a compensatory mechanism in which the mutant responds to increased levels of oxidative damage in mtDNA in the gas1 strain. Further exploration of this idea awaits data from the gas-1 MA lines. 


\section{References}

Ameur, A., Stewart, J. B., Freyer, C., Hagström, E., Ingman, M., Larsson, N.-G., \& Gyllensten, U. (2011). Ultra-deep sequencing of mouse mitochondrial DNA: mutational patterns and their origins. PLoS Genetics, 7(3), e1002028. http://doi.org/10.1371/journal.pgen.1002028

Azevedo, L., Carneiro, J., van Asch, B., Moleirinho, A., Pereira, F., \& Amorim, A. (2009). Epistatic interactions modulate the evolution of mammalian mitochondrial respiratory complex components. BMC Genomics, 10, 266. http://doi.org/10.1186/1471-2164-10-266

Azevedo, R. B. R., Keightley, P. D., Laurén-Määttä, C., Vassilieva, L. L., Lynch, M., \& Leroi, A. M. (2002). Spontaneous mutational variation for body size in Caenorhabditis elegans. Genetics, 162(2), 755-65. Retrieved from http://www.pubmedcentral.nih.gov/articlerender.fcgi?artid=1462287\&tool=pmcentr ez\&rendertype $=$ abstract

Baer, C. F., Miyamoto, M. M., \& Denver, D. R. (2007). Mutation rate variation in multicellular eukaryotes: causes and consequences. Nature Reviews. Genetics, 8(8), 619-31. http://doi.org/10.1038/nrg2158

Baer, C. F., Phillips, N., Ostrow, D., Avalos, A., Blanton, D., Boggs, A., ... Mezerhane, E. (2006). Cumulative effects of spontaneous mutations for fitness in Caenorhabditis: role of genotype, environment and stress. Genetics, 174(3), 138795. http://doi.org/10.1534/genetics. 106.061200

Baer, C. F., Shaw, F., Steding, C., Baumgartner, M., Hawkins, A., Houppert, A., ... Lynch, M. (2005). Comparative evolutionary genetics of spontaneous mutations affecting fitness in rhabditid nematodes. Proceedings of the National Academy of Sciences of the United States of America, 102(16), 5785-90. http://doi.org/10.1073/pnas.0406056102

Ballard, J. W. O., \& Whitlock, M. C. (2004). The incomplete natural history of mitochondria. Molecular Ecology, 13(4), 729-744. http://doi.org/10.1046/j.1365294X.2003.02063.x

Brand, M. D. (2000). Uncoupling to survive? The role of mitochondrial inefficiency in ageing. Experimental Gerontology, 35, 811-820.

Brownlee, M. (2001). Biochemistry and molecular cell biology of diabetic complications. Nature, 414(6865), 813-20.

Burggren, W. W., \& Freeman, S. (2011). Biological Science (4th ed.). Boston: Benjamin Cummings.

Busuttil, R. a., Rubio, M., Doll, M. E. T., Campisi, J., \& Vijg, J. (2003). Oxygen accelerates the accumulation of mutations during the senescence and immortalization of murine cells in culture. Aging Cell, 2(6), 287-294. http://doi.org/10.1046/j.1474-9728.2003.00066.x

Colegrave, N., \& Buckling, A. (2005). Microbial experiments on adaptive landscapes. BioEssays : News and Reviews in Molecular, Cellular and Developmental Biology, 27(11), 1167-73. http://doi.org/10.1002/bies.20292 
Colegrave, N., \& Collins, S. (2008). Experimental evolution: experimental evolution and evolvability. Heredity, 100(5), 464-70. http://doi.org/10.1038/sj.hdy.6801095

Collins, J. J., Huang, C., Hughes, S., \& Kornfeld, K. (2007). The measurement and analysis of age-related changes in Caenorhabditis elegans. WormBook: The Online Review of C. Elegans Biology, (314), 1-21. http://doi.org/10.1895/wormbook.1.137.1

Consortium, I. H. G. S. (2004). Finishing the euchromatic sequence of the human genome. Nature, 431, 931-45. Retrieved from http://www.ncbi.nlm.nih.gov/pubmed/15704464

Craige, B., Salazar, G., \& Faundez, V. (2008). Phosphatidylinositol-4-Kinase Type II Alpha Contains an AP-3 - sorting Motif and a Kinase Domain That Are Both Required for Endosome Traffic. Molecular Biology of the Cell, 19(April), 14151426. http://doi.org/10.1091/mbc.E07

Crick, F. (1981). Life Itself: Its Origin and Nature. New York: Simon \& Schuster.

Crow, J., \& Kimura, M. (1970). An introduction to population genetics theory.

Davies, E. K., Peters, A. D., \& Keightley, P. D. (1999). High Frequency of Cryptic Deleterious Mutations in Caenorhabditis elegans. Science, 285(September), 17481751.

Denver, D. R. (2000). High Direct Estimate of the Mutation Rate in the Mitochondrial Genome of Caenorhabditis elegans. Science, 289(5488), 2342-2344. http://doi.org/10.1126/science.289.5488.2342

Denver, D. R., Dolan, P. C., Wilhelm, L. J., Sung, W., Lucas-Lledó, J. I., Howe, D. K., ... Baer, C. F. (2009). A genome-wide view of Caenorhabditis elegans basesubstitution mutation processes. Proceedings of the National Academy of Sciences of the United States of America, 106(38), 16310-4. http://doi.org/10.1073/pnas.0904895106

Denver, D. R., Feinberg, S., Steding, C., Durbin, M., \& Lynch, M. (2006). The relative roles of three DNA repair pathways in preventing Caenorhabditis elegans mutation accumulation. Genetics, 174(1), 57-65. http://doi.org/10.1534/genetics.106.059840

Denver, D. R., Howe, D. K., Wilhelm, L. J., Palmer, C. A., Anderson, J. L., Stein, K. C., ... Estes, S. (2010). Selective sweeps and parallel mutation in the adaptive recovery from deleterious mutation in Caenorhabditis elegans. Genome Research, 20(541), 1663-1671. http://doi.org/10.1101/gr.108191.110.20

Denver, D. R., Morris, K., Lynch, M., \& Thomas, W. K. (2004). High mutation rate and predominance of insertions in the Caenorhabditis elegans nuclear genome. Nature, 430(7000), 679-82. http://doi.org/10.1038/nature02697

Denver, D. R., Wilhelm, L. J., Howe, D. K., Gafner, K., Dolan, P. C., \& Baer, C. F. (2012). Variation in base-substitution mutation in experimental and natural lineages of Caenorhabditis nematodes. Genome Biology and Evolution, 4(4), 513-22. http://doi.org/10.1093/gbe/evs028

Dowling, D. K., Friberg, U., \& Lindell, J. (2008). Evolutionary implications of nonneutral mitochondrial genetic variation. Trends in Ecology \& Evolution, 23(10), 546-54. http://doi.org/10.1016/j.tree.2008.05.011 
Ericson, N. G., Kulawiec, M., Vermulst, M., Sheahan, K., Sullivan, J. O., Salk, J. J., \& Bielas, J. H. (2012). Decreased Mitochondrial DNA Mutagenesis in Human Colorectal Cancer. PLoS Genetics, 8(6), e1002689. http://doi.org/10.1371/journal.pgen.1002689

Estes, S., Ajie, B. C., Lynch, M., \& Phillips, P. C. (2005). Spontaneous mutational correlations for life-history, morphological and behavioral characters in Caenorhabditis elegans. Genetics, 170(2), 645-53. http://doi.org/10.1534/genetics.104.040022

Estes, S., Coleman-Hulbert, A. L., Hicks, K. a, de Haan, G., Martha, S. R., Knapp, J. B., ... Denver, D. R. (2011). Natural variation in life history and aging phenotypes is associated with mitochondrial DNA deletion frequency in Caenorhabditis briggsae. BMC Evolutionary Biology, 11(1), 11. http://doi.org/10.1186/1471-2148-11-11

Estes, S., \& Phillips, P. C. (2006). Variation in pleiotropy and the mutational underpinnings of the G-matrix. Evolution, 60, 2655.

Estes, S., Phillips, P. C., Denver, D. R., Thomas, W. K., \& Lynch, M. (2004). Mutation accumulation in populations of varying size: the distribution of mutational effects for fitness correlates in Caenorhabditis elegans. Genetics, 166(3), 1269-79. Retrieved from http://www.pubmedcentral.nih.gov/articlerender.fcgi?artid=1470770\&tool=pmcentr ez\&rendertype $=$ abstract

Estes, S., \& Teotonio, H. (2009). The experimental study of reverse evolution. Experimental Evolution: Concepts, Methods, and Applications of Selection Experiments, 135-155.

Evans, E. A., Chen, W. C., \& Tan, M. (2008). The DAF-2 Insulin-like Signaling Pathway Independently Regulated Aging and Immunity in C. elegans. Aging Cell, 7(6), 879893. http://doi.org/10.1111/j.1474-9726.2008.00435.x.The

Falk, M., Zhang, Z., Rosenjack, J., Nissim, I., Daikhin, E., Sedensky, M., ... Morgan, P. (2008). Metabolic pathway profiling of mitochondrial respiratory chain mutants in C. elegans. Mol Genet Metab, 93(4), 388-397.

Gentry, K. R., Steele, L. M., Sedensky, M. M., \& Morgan, P. G. (2013). Early Developmental Exposure to Volatile Anesthetics Causes Behavioral Defects in Caenorhabditis elegans. Anesth Analg., 116(1), 185-189. http://doi.org/10.1213/ANE.0b013e31826d37c5.Early

Groenewoud, M. J., \& Zwartkruis, F. J. T. (2013). Rheb and mammalian target of rapamycin in mitochondrial homoeostasis. Open Biology, 3(12), 130185. http://doi.org/10.1098/rsob.130185

Haag-Liautard, C., Dorris, M., Maside, X., Macaskill, S., Halligan, D. L., Houle, D., ... Keightley, P. D. (2007). Direct estimation of per nucleotide and genomic deleterious mutation rates in Drosophila. Nature, 445(7123), 82-5. http://doi.org/10.1038/nature05388

Halligan, D. L., \& Keightley, P. D. (2009). Spontaneous Mutation Accumulation Studies in Evolutionary Genetics. Annual Review of Ecology, Evolution, and Systematics, 40(1), 151-172. http://doi.org/10.1146/annurev.ecolsys.39.110707.173437 
Hancock, J. T., Desikan, R., \& Neill, S. J. (2001). Role of reactive oxygen species in cell signalling pathways. Biochemical and Biomedical Aspects of Oxidative Modification, 345-350.

Handel, A., Regoes, R. R., \& Antia, R. (2006). The role of compensatory mutations in the emergence of drug resistance. PLoS Computational Biology, 2(10), e137. http://doi.org/10.1371/journal.pcbi.0020137

Harrison, J. S., \& Burton, R. S. (2006). Tracing hybrid incompatibilities to single amino acid substitutions. Molecular Biology and Evolution, 23(3), 559-64. http://doi.org/10.1093/molbev/msj058

Hartman, P., Ishii, N., Kayser, E., Morgan, P., \& Sedensky, M. (2001). Mitochondrial mutations differentially affect aging, mutability and anesthetic sensitivity in Caenorhabditis elegans. Mech Ageing Dev, 122(11), 1187-201.

Heilbron, K., Toll-Riera, M., Kojadinovic, M., \& MacLean, R. C. (2014). Fitness is strongly influenced by rare mutations of large effect in a microbial mutation accumulation experiment. Genetics, 197(3), 981-90. http://doi.org/10.1534/genetics.114.163147

Hicks, K. a, Howe, D. K., Leung, A., Denver, D. R., \& Estes, S. (2012). In vivo quantification reveals extensive natural variation in mitochondrial form and function in Caenorhabditis briggsae. PloS One, 7(8), e43837. http://doi.org/10.1371/journal.pone.0043837

Hirst, J., Carroll, J., Fearnley, I. M., Shannon, R. J., \& Walker, J. E. (2003). The nuclear encoded subunits of complex I from bovine heart mitochondria. Biochimica et Biophysica Acta (BBA) - Bioenergetics, 1604(3), 135-150. http://doi.org/10.1016/S0005-2728(03)00059-8

Hope, I. (Ed.). (1999). C. elegans: A Practical Approach (1st ed., pp. 51-67). Leeds: Oxford University Press.

Hu, P. J. (2007). Dauer. WormBook: The Online Review of C. Elegans Biology, (734), 119. http://doi.org/10.1895/wormbook.1.144.1

Itsara, L. S., Kennedy, S. R., Fox, E. J., Yu, S., Hewitt, J. J., Sanchez-Contreras, M., ... Pallanck, L. J. (2014). Oxidative stress is not a major contributor to somatic mitochondrial DNA mutations. PLoS Genetics, 10(2), e1003974. http://doi.org/10.1371/journal.pgen.1003974

Jones, A. G., Arnold, S. J., \& Bürger, R. (2007). The mutation matrix and the evolution of evolvability. Evolution; International Journal of Organic Evolution, 61(4), 727 45. http://doi.org/10.1111/j.1558-5646.2007.00071.x

Joyner-Matos, J., Bean, L. C., Richardson, H. L., Sammeli, T., \& Baer, C. F. (2011). No evidence of elevated germline mutation accumulation under oxidative stress in Caenorhabditis elegans. Genetics, 189(4), 1439-47. http://doi.org/10.1534/genetics.111.133660

Joyner-Matos, J., Hicks, K. a, Cousins, D., Keller, M., Denver, D. R., Baer, C. F., \& Estes, S. (2013). Evolution of a higher intracellular oxidizing environment in Caenorhabditis elegans under relaxed selection. PloS One, 8(6), e65604. http://doi.org/10.1371/journal.pone.0065604 
Kayser, E. B., Morgan, P. G., Hoppel, C. L., \& Sedensky, M. M. (2001). Mitochondrial expression and function of GAS-1 in Caenorhabditis elegans. The Journal of Biological Chemistry, 276(23), 20551-8. http://doi.org/10.1074/jbc.M011066200

Keightley, P. D., \& Bataillon, T. M. (2000). Multigeneration Maximum-Likelihood Analysis Applied to in Caenorhabditis elegans. Genetics, 154, 1193-1201.

Keightley, P. D., Trivedi, U., Thomson, M., Oliver, F., Kumar, S., \& Blaxter, M. L. (2009). Analysis of the genome sequences of three Drosophila melanogaster spontaneous mutation accumulation lines. Genome Research, 19(7), 1195-201. http://doi.org/10.1101/gr.091231.109

Kenyon, C. (2011). The first long-lived mutants: discovery of the insulin/IGF-1 pathway for ageing. Philosophical Transactions of the Royal Society of London. Series B, Biological Sciences, 366(1561), 9-16. http://doi.org/10.1098/rstb.2010.0276

Kondo, M., Senoo-Matsuda, N., Yanase, S., Ishii, T., Hartman, P., \& Ishii, N. (2005). Effect of oxidative stress on translocation of DAF-16 in oxygen-sensitive mutants, mev-1 and gas-1 of Carnorhabditis elegans. Mech Ageing Dev, 126(6-7), 637-41.

Kondrashov, F. a, \& Kondrashov, A. S. (2010). Measurements of spontaneous rates of mutations in the recent past and the near future. Philosophical Transactions of the Royal Society of London. Series B, Biological Sciences, 365(1544), 1169-76. http://doi.org/10.1098/rstb.2009.0286

Korshunov, S. S., Skulachev, V. P., \& Starkov, A. a. (1997). High protonic potential actuates a mechanism of production of reactive oxygen species in mitochondria. FEBS Letters, 416(1), 15-18. http://doi.org/10.1016/S0014-5793(97)01159-9

Lande, R. (1994). Risk of Population Extinction from Fixation of New Deleterious Mutations A. Evolution, 48(5), 1460-1469.

Lane, N. (2011). Mitonuclear match: optimizing fitness and fertility over generations drives ageing within generations. BioEssays : News and Reviews in Molecular, Cellular and Developmental Biology, 33(11), 860-9. http://doi.org/10.1002/bies.201100051

Laplante, M., \& Sabatini, D. M. (2009). mTOR signaling at a glance. Journal of Cell Science, 122(Pt 20), 3589-94. http://doi.org/10.1242/jcs.051011

Lemire, B. (2005). Mitochondrial genetics. WormBook: The Online Review of C. Elegans Biology, 1-10. http://doi.org/10.1895/wormbook.1.25.1

Lenaz, G., Baracca, A., Fato, R., Genova, M. L., \& Solaini, G. (2006). New Insights Into Structure and Function of Mitochondria and Their Role in Aging and Disease. Antioxidants \& Redox Signaling3\&4, 8(3 \& 4), 417-435.

Lenaz, G., Fato, R., Genova, M. L., Bergamini, C., Bianchi, C., \& Biondi, A. (2006). Mitochondrial Complex I: structural and functional aspects. Biochimica et Biophysica Acta, 1757(9-10), 1406-20. http://doi.org/10.1016/j.bbabio.2006.05.007

Levin, B. R., \& Walker, N. (2000). Compensatory Mutations, Antibiotic Resistance and the Population Genetics of Adaptive Evolution in Bacteria. Genetics, 154, 985-997.

Lynch, M., Blanchard, J., Houle, D., Kibota, T., Schultz, S., \& Vassilieva, L. (1999). Perspective: Spontaneous Deleterious Mutation. Evolution, 53(3), 645-663. 
Lynch, M., Conery, J., \& Burger, R. (1995). Mutation Accumulation and the Extinction of Small Populations. The American Naturalist, 146(4), 489-518.

Lynch, M., Koskella, B., \& Schaack, S. (2006). Mutation pressure and the evolution of organelle genomic architecture. Science (New York, N.Y.), 311(5768), 1727-30. http://doi.org/10.1126/science.1118884

Lynch, M., Sung, W., Morris, K., Coffey, N., Landry, C. R., Dopman, E. B., ... Thomas, W. K. (2008). A genome-wide view of the spectrum of spontaneous mutations in yeast. $P N A S, 105(27), 9272-9277$.

Lynch, M., \& Walsh, J. B. (1997). Genetics and Analysis of Quantitative Characters (p. 980). Sinauer Associations.

Morgan, P. G., Kayser, E.-B., \& Sedensky, M. M. (2007). C. elegans and volatile anesthetics. WormBook: The Online Review of C. Elegans Biology, 1-11. http://doi.org/10.1895/wormbook.1.140.1

Murphy, C. T., \& Hu, P. J. (2013). Insulin/insulin-like growth factor signaling in C . elegans. WormBook: The Online Review of C. Elegans Biology, 1-43. http://doi.org/10.1895/wormbook.1.

Osada, N., \& Akashi, H. (2011). Mitochondrial - Nuclear Interactions and Accelerated Compensatory Evolution: Evidence from the Primate Cytochrome c Oxidase Complex. Mol. Biol. Evol., (1973), 1-10. http://doi.org/10.1093/molbev/msr211

Ossowski, S., Schneeberger, K., Lucas-lledó, J. I., Clark, R. M., Shaw, R. G., Weigel, D., \& Lynch, M. (2010). The Rate and Molecular Spectrum of Spontaneous Mutations in Arabidopsis thaliana. Science, 327(5961), 1-9. http://doi.org/10.1126/science.1180677.The

Raha, S., \& Robinson, B. (2000). Mitochondria, oxygen free radicals, disease and ageing. Biochem Sci, 25, 502-508.

Rand, D. M. (2008). Mitigating mutational meltdown in mammalian mitochondria. PLoS Biology, 6(2), e35. http://doi.org/10.1371/journal.pbio.0060035

Rodriguez, M., Snoek, L. B., De Bono, M., \& Kammenga, J. E. (2013). Worms under stress: C. elegans stress response and its relevance to complex human disease and aging. Trends in Genetics : TIG, 29(6), 367-74. http://doi.org/10.1016/j.tig.2013.01.010

Ruckenstuhl, C., Büttner, S., Carmona-Gutierrez, D., Eisenberg, T., Kroemer, G., Sigrist, S. J., ... Madeo, F. (2009). The Warburg effect suppresses oxidative stress induced apoptosis in a yeast model for cancer. PloS One, 4(2), e4592. http://doi.org/10.1371/journal.pone.0004592

Samuelson, A. V, Carr, C. E., \& Ruvkun, G. (2007). Gene activities that mediate increased life span of C . elegans insulin-like signaling mutants. Genes and Development, 21, 2976-2994. http://doi.org/10.1101/gad.1588907.1993

Saxer, G., Havlak, P., Fox, S. a, Quance, M. a, Gupta, S., Fofanov, Y., ... Queller, D. C. (2012). Whole genome sequencing of mutation accumulation lines reveals a low mutation rate in the social amoeba Dictyostelium discoideum. PloS One, 7(10), e46759. http://doi.org/10.1371/journal.pone.0046759 
Schaefer, A. M., Taylor, R. W., Turnbull, D. M., \& Chinnery, P. F. (2004). The epidemiology of mitochondrial disorders--past, present and future. Biochimica et Biophysica Acta, 1659(2-3), 115-20. http://doi.org/10.1016/j.bbabio.2004.09.005

Schultz, S. T., \& Lynch, M. (1997). Mutation and Extinction: The Role of Variable Mutational Effects, Synergistic Epistasis, Beneficial Mutations, and Degree of Outcrossing. Evolution, 51(5), 1363-1371.

Senoo-Matsuda, N., Yasuda, K., Tsuda, M., Ohkubo, T., Yoshimura, S., Nakazawa, H., ... Ishii, N. (2001). A defect in the cytochrome b large subunit in complex II causes both superoxide anion overproduction and abnormal energy metabolism in Caenorhabditis elegans. The Journal of Biological Chemistry, 276(45), 41553-8. http://doi.org/10.1074/jbc.M104718200

Smith, S. W., Latta, L. C., Denver, D. R., \& Estes, S. (2014). Endogenous ROS levels in C. elegans under exogenous stress support revision of oxidative stress theory of lifehistory tradeoffs. BMC Evolutionary Biology, 14, 161. http://doi.org/10.1186/s12862-014-0161-8

Stiernagle, T. (2006). Maintenance of C. elegans. WormBook: The Online Review of C. Elegans Biology, (1999), 1-11. http://doi.org/10.1895/wormbook.1.101.1

Vassilieva, L. L., Hook, A. M., Lynch, M., \& Aug, N. (2000). The Fitness Effects of Spontaneous Mutations in Caenorhabdits elegans. Evolution, 54(4), 1234-1246.

Vassilieva, L. L., \& Lynch, M. (1999). The Rate of Spontaneous Mutation for LifeHistory Traits in Caenorhabditis elegans. Genetics, 151, 119-29.

Warburg, O. (1956). On respiratory impairment in cancer cells. Science, 124(3215), 26970 .

Westermann, B. (2010). Mitochondrial dynamics in model organisms: What yeasts, worms, and flies have taught us about fusion and fission of mitochondria. Seminars in Cell \& Developmental Biology, 21, 542-549.

Yu, E., Calvert, P. a, Mercer, J. R., Harrison, J., Baker, L., Figg, N. L., ... Bennett, M. R. (2013). Mitochondrial DNA damage can promote atherosclerosis independently of reactive oxygen species through effects on smooth muscle cells and monocytes and correlates with higher-risk plaques in humans. Circulation, 128(7), 702-12.

http://doi.org/10.1161/CIRCULATIONAHA.113.002271 